\title{
Essentially Normal Composition Operators
}

\author{
Mor Katz \\ Charlottesville, VA
}

MS., University of Virginia, 2012

BS., Tel-Aviv University, 2006

A Dissertation presented to the Graduate Faculty of the University of Virginia in Candidacy for the Degree of Doctor of Philosophy

Department of Mathematics

University of Virginia

December, 2013 


\begin{abstract}
In this dissertation we prove a simple criterion for essential normality of composition operators $C_{\varphi}$ induced by maps $\varphi$ in a large class of functions. Additionally, we construct essentially normal composition operators which have arbitrary even order of contact with the unit circle at one point. To do so, we rely on results from three distinct areas. We use results and techniques of Agler-Lykova-Young to construct rational analytic self-maps of the unit disk with specified Taylor coefficients at a given boundary point. This allows us to decompose a composition operator modulo the ideal of compact operators into a sum of rationally induced composition operators based on results of Kriete-Moorhouse. The adjoint of each rationally induced composition operator is then studied using results and ideas of Bourdon-Shapiro. Essential normality is then characterized, beginning with a single summand in the decomposition and continuing to the more general case. Finally, we construct essentially normal composition operators which have arbitrary even order of contact with the unit circle at one point.
\end{abstract}




\section{Acknowledgments}

I would like to thank my advisor, Thomas Kriete, for his continuous guidance and support, his spot on intuition, his attention to detail, and his willingness to treat every question as if it were a good one.

I am also thankful to the members of my committee, Paul Bourdon, Barbara MacCluer, Olivier Pfister and David Sherman, for their time and helpful comments and suggestions which all helped improve this thesis. In particular, I would like to thank Paul Bourdon for his list of thirty nine comments ranging from typos to insightful suggestions for improvement.

Finally, thank you to my family - my husband Arnie, my parents Natan and Dorit, and my sisters Shaked and Yasmin, for supporting me throughout the past five years. A special thanks goes to my daughter, Danielle, for decorating so many of my drafts with orange markers. 


\section{Contents}

1 Introduction 1

2 Preliminary Material $\quad 4$

2.1 Bounded Operators on a Hilbert Space . . . . . . . . . . . . . 4

2.2 The Hardy Space $H^{2}$, Composition Operators and Toeplitz Operators 6

2.3 More About Composition Operators . . . . . . . . . . . . . 7

2.4 Order of Contact . . . . . . . . . . . . . . . . . 12

2.5 Rational Functions . . . . . . . . . . . . . . . . . 15

3 The Boundary Carathéodory-Fejér Problem 18

3.1 The Boundary Carathéodory-Fejér Problem _ . . . . . . . . . . . . 19

3.2 Reduction and Augmentation . . . . . . . . . . . . . . 21

3.3 Parametrization of Solutions to the Contact-n Case . . . . . . . . . . 24

3.4 Rational Functions with Specified Taylor Coefficients . . . . . . . . . 29 
4 Relations In The Calkin Algebra $\quad 34$

4.1 The Class of Functions $\mathcal{A}$. . . . . . . . . . . . . . . . . . . 34

4.2 Linear Relations for Composition Operators in the Calkin Algebra . . 37

4.3 Weighted Composition Operators and Adjoints in the Calkin Algebra 42

5 Adjoint Formula for Rationally Induced Composition Operators 49

5.1 From Pointwise Formula To Operator Equation . . . . . . . . . . . . 49

5.2 The Basic Case . . . . . . . . . . . . . . . . . 55

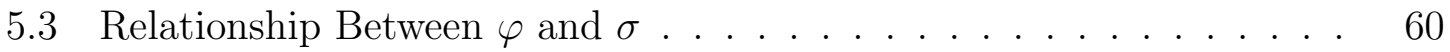

$\begin{array}{lll}6 & \text { Essential normality } & 67\end{array}$

6.1 Essential Normality For Basic Composition Operators . . . . . . . . . 67

6.2 General Essential Normality . . . . . . . . . . . . . . . . 71

6.3 Construction of Essentially Normal Composition Operators . . . . . . 78 


\section{Chapter 1}

\section{Introduction}

For $\varphi$ an analytic self-map of the unit disk $\mathbb{D}$, the composition operator $C_{\varphi}: f \rightarrow f \circ \varphi$ induced by $\varphi$ is a bounded operator on the Hardy space $H^{2}$. Composition operators have been widely studied and many results can be found in [13] and [25].

Recently, several authors have investigated $C^{*}$-algebras generated by one or more composition operators. One aim in studying such $C^{*}$-algebras is to understand the properties of the operators they contain. In particular, there is much interest in determining the spectrum of algebraic combinations of composition operators and their adjoints.

For $C_{\varphi}$ an essentially normal composition operator, the quotient algebra of the $C^{*}$-algebra generated by $C_{\varphi}$ by the ideal of the compact operators $\mathcal{K}$ is commutative, and so spectral theory in this quotient algebra can in principle be understood via Gelfand Theory. In [6], Bourdon-Levi-Narayan-Shapiro characterize the class of nontrivially essentially normal composition operators induced by linear fractional maps and provide additional examples of essentially normal composition operators induced by maps which have order of contact 2 with $\partial \mathbb{D}$ at one point. To the best of the 
author's knowledge, no non-trivially essentially normal composition operators having order of contact $n>2$ with the unit circle were known prior to the present work.

In this dissertation we prove a simple criterion for essential normality of composition operators induced by maps $\varphi$ in a large class of functions. Additionally, we construct essentially normal composition operators which have arbitrary even order of contact with the unit circle at one point. To do so, we rely on results from three distinct areas, presented in Chapters 3-5 after a review of preliminary material in Chapter 2 .

In Chapter 3 we discuss a boundary version of the Carathéodory-Fejér problem studied by Agler-Lykova-Young in $[1,2]$, of determining whether a given finite sequence of complex numbers comprises the initial Taylor coefficients of an analytic self-map of the upper half-plane, $\mathbb{H}$, at a boundary point. We review several of the results and techniques used in $[1,2]$, then derive similar results for a slightly different case of interest. We apply this to obtain a parametrization of all analytic self-maps of $\mathbb{H}$ having specified order of contact with the real line at 0 , and to construct rational analytic self-maps of $\mathbb{H}$ that are analytic at 0 and have specified Taylor coefficients there.

In Chapter 4 we review some definitions and results of Kriete-Moorhouse [19] regarding compactness of linear combinations of composition operators. We then apply our results from Chapter 3 to obtain a decomposition of a composition operator modulo $\mathcal{K}$ into a sum of composition operators induced by rational functions. We 
define basic functions as rational self-maps of the unit disk satisfying some additional properties, and prove a version of the decomposition theorem with basic functions. Additionally, we review a result from [19] regarding weighted composition operators in the Calkin algebra and use this result in the proof of a similar result concerning weighted adjoints of composition operators.

In Chapter 5 we use the pointwise formula for the adjoint of a rationally induced composition operator $C_{\varphi}^{*}$ (introduced in $\left.[12,15]\right)$ to obtain an operator formula for $C_{\psi} C_{\varphi}^{*}$ where $\psi$ satisfies some conditions. We use a variant of the formula which appears in Bourdon-Shapiro [7] and ideas from [7] to reduce our formula modulo $\mathcal{K}$ for the special case where $\varphi$ is a basic function.

Finally, in Chapter 6 we investigate essential normality. We begin by proving a necessary and sufficient condition for essential normality for the case where the inducing map $\varphi$ is a basic function. We then decompose $C_{\varphi}$ into a sum of basic composition operators modulo $\mathcal{K}$ and show that essential normality can only occur when this sum consists of a single term. Lastly, using the parametrization of functions with given order of contact proved in Chapter 3, we construct essentially normal composition operators which have arbitrary even order of contact with the unit circle at one point. 


\section{Chapter 2}

\section{Preliminary Material}

\subsection{Bounded Operators on a Hilbert Space}

For any Hilbert space $\mathcal{H}$, we denote the space of all bounded linear operators from $\mathcal{H}$ to $\mathcal{H}$ by $\mathcal{B}(\mathcal{H})$. The operator norm on $\mathcal{B}(\mathcal{H})$ is defined by

$$
\|T\|=\sup \left\{\|T h\|: h \in \mathcal{H},\|h\|_{\mathcal{H}} \leq 1\right\}
$$

for all $T \in \mathcal{B}(\mathcal{H})$, and $\mathcal{B}(\mathcal{H})$ is complete in this norm.

We say that an operator $T \in \mathcal{B}(\mathcal{H})$ is finite rank if its range is finite dimensional. An operator $T$ is compact if the following equivalent conditions hold.

Proposition 2.1.1. [23, Theorem 3.3.3] The following conditions are equivalent.

1. $T$ is in the closure of the set of finite rank operators in $\mathcal{B}(\mathcal{H})$

2. The closure of the image of the unit ball of $H$ under $T$ is compact in $\mathcal{H}$

3. Each net in the unit ball of $\mathcal{H}$ has a subnet whose image under $T$ converges in $\mathcal{H}$ 
Note that all finite rank operators are compact. Additionally, from the third condition in Proposition 2.1.1 it follows that:

Proposition 2.1.2. $T \in \mathcal{B}(\mathcal{H})$ is compact and $S \in \mathcal{B}(\mathcal{H})$ satisfies $\|S h\| \leq\|T h\|$ for all $h \in \mathcal{H}$, then $S$ is compact as well.

We denote the collection of all compact operators on $\mathcal{H}$ by $\mathcal{K}$ and note that this collection forms a closed, two-sided ideal in $\mathcal{B}(\mathcal{H})$. The quotient algebra $\mathcal{B}(\mathcal{H}) / \mathcal{K}$ is called the Calkin algebra $[4,23]$. If $T-S \in \mathcal{K}$, i.e., $T$ and $S$ are in the same coset in the Calkin algebra, we write

$$
T \equiv S \quad(\bmod \mathcal{K})
$$

For each operator $T \in \mathcal{B}(\mathcal{H})$ there exists a unique operator $T^{*} \in \mathcal{B}(\mathcal{H})$, called the adjoint of $T$, that satisfies

$$
\langle T g, h\rangle=\left\langle g, T^{*} h\right\rangle
$$

for all $g, h \in \mathcal{H}$. An operator $T$ is normal if it commutes with its adjoint, that is, $T T^{*}=T^{*} T$, and essentially normal if the self-commutator $\left[T, T^{*}\right]=T T^{*}-T^{*} T$ is compact, that is,

$$
T^{*} T \equiv T T^{*} \quad(\bmod \mathcal{K})
$$




\subsection{The Hardy Space $H^{2}$, Composition Operators and Toeplitz Operators}

The Hardy space of the disk, $H^{2}:=H^{2}(\mathbb{D})$, is the space of all analytic functions $f$ on

$\mathbb{D}$ whose power series $f(z)=\sum_{n=0}^{\infty} a_{n} z^{n}$ satisfy

$$
\|f\|_{H^{2}}^{2}:=\sum_{n=0}^{\infty}\left|a_{n}\right|^{2}<\infty .
$$

The Hardy space is a Hilbert space under the inner product

$$
\left\langle\sum_{n=0}^{\infty} a_{n} z^{n}, \sum_{n=0}^{\infty} b_{n} z^{n}\right\rangle_{H^{2}}:=\sum_{n=0}^{\infty} a_{n} \overline{b_{n}} .
$$

More information about the Hardy space can be found in [13].

We can identify $f(z)=\sum_{0}^{\infty} a_{n} z^{n}$ in $H^{2}$ with the boundary function $f\left(e^{i \theta}\right)=$ $\sum_{0}^{\infty} a_{n} e^{i n \theta}$ in $H^{2}(\partial \mathbb{D})$. Note that

$$
f\left(e^{i \theta}\right)=\lim _{r \rightarrow 1} f\left(r e^{i \theta}\right)
$$

for almost every $\theta[21]$.

For $\varphi$ in $L^{\infty}(\partial \mathbb{D})$, we define the multiplication operator $M_{\varphi}$ acting on $L^{2}(\partial \mathbb{D})$ by

$$
\left(M_{\varphi} f\right)\left(e^{i \theta}\right)=\varphi\left(e^{i \theta}\right) f\left(e^{i \theta}\right)
$$

and the Toeplitz operator $T_{\varphi}$ acting on $H^{2}(\partial \mathbb{D})$ by $T_{\varphi}=\left.P M_{\varphi}\right|_{H^{2}}$, where $P$ is the projection of $L^{2}(\partial \mathbb{D})$ onto $H^{2}(\partial \mathbb{D})$.

For $\varphi$ an analytic self map of $\mathbb{D}$, we define the composition operator $C_{\varphi}$ on $H^{2}$ by

$$
\mathbb{C}_{\varphi} f=f \circ \varphi
$$


By Littlewood's subordination principle, $C_{\varphi}$ is a bounded linear operator on $H^{2}$ [21]. The space $H^{\infty}$ consisting of all the functions that are analytic and bounded on $\mathbb{D}$ arises in the study of operators on $H^{2} . H^{\infty}$ is a Banach algebra with pointwise operations and the sup norm, and is contained in $H^{2}$.

\subsection{More About Composition Operators}

\subsubsection{Compactness for Composition Operators}

Compactness of the composition operator $C_{\varphi}$ is closely related to the boundary behavior of $\varphi$. The following two results suggest that $C_{\varphi}$ is compact when $\varphi(z)$ does not get too close to the boundary too often.

Proposition 2.3.1. [25, $p$ 23] If $\|\varphi\|_{\infty}<1$ then $C_{\varphi}$ is compact.

Proposition 2.3.2. [25, $p$ 32] If the set $\{\zeta \in \partial \mathbb{D}:|\varphi(\zeta)|=1\}$ has positive Lebesgue measure then $C_{\varphi}$ is not compact

In order to state additional results relating the boundary behavior of $\varphi$ and compactness of $C_{\varphi}$, we introduce the notion of non-tangential approach regions, angular derivatives and the Denjoy-Wolff point.

For $\zeta \in \partial \mathbb{D}$ and $\alpha>1$ we define the non-tangential approach region at $\zeta$ by

$$
\Gamma_{\alpha}(\zeta)=\{z \in \mathbb{D}:|z-\zeta|<\alpha(1-|z|)\} .
$$


Figure 2.1: A typical non-tangential approach region $\left(\alpha=3, \zeta=e^{\frac{\pi}{4} i}\right)$

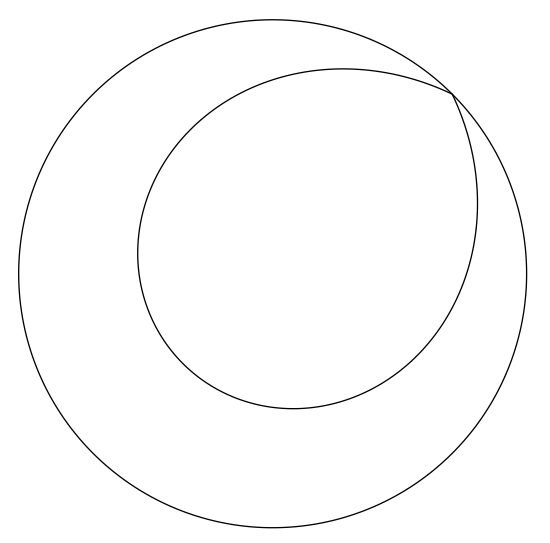

We say a function $f$ on $\mathbb{D}$ has non-tangential limit $L \in \mathbb{C}$ at $\zeta$ and write

$$
\angle \lim _{z \rightarrow \zeta} f(z)=L
$$

if $f(z) \rightarrow L$ as $z \rightarrow \zeta$ along any path contained in a non-tangential approach region at $\zeta$.

Let $\varphi$ be an analytic self-map of $\mathbb{D}$. Then $\varphi$ has a (finite) angular derivative at $\zeta$ in $\partial \mathbb{D}$ provided $\varphi(\zeta)$, the non-tangential limit of $\varphi$ at $\zeta$, exists and has modulus one, and

$$
\varphi^{\prime}(\zeta):=\angle \lim _{z \rightarrow \zeta} \frac{\varphi(z)-\varphi(\zeta)}{z-\zeta}
$$

exists as a finite complex number. If the angular derivative $\varphi^{\prime}(\zeta)$ fails to exist, we write $\left|\varphi^{\prime}(\zeta)\right|=\infty$

Theorem 2.3.3 (Julia Carathéodory [13]). For $\varphi$ an analytic self-map of $\mathbb{D}$ and $\zeta \in \partial \mathbb{D}$, the following are equivalent

$$
\text { 1. } d(\zeta)=\liminf _{z \rightarrow \zeta} \frac{1-|\varphi(z)|}{1-|z|}<\infty
$$


2. $\varphi$ has finite angular derivative $\varphi^{\prime}(\zeta)$ at $\zeta$

3. Both $\varphi$ and $\varphi^{\prime}$ have (finite) non-tangential limits at $\zeta$, with $|\eta|=1$ for $\eta=\lim _{r \rightarrow 1} \varphi(r \zeta)$.

Moreover, when these conditions hold, we have $\lim _{r \rightarrow 1} \varphi^{\prime}(r \zeta)=\varphi^{\prime}(\zeta)=d(\zeta) \bar{\zeta} \eta$ and $d(\zeta)=\angle \lim _{z \rightarrow \zeta} \frac{1-|\varphi(z)|}{1-|z|}$.

The angular derivative criterion for compactness is given by the following result.

Proposition 2.3.4. [25, $p$ 57] Suppose $\varphi$ is an analytic self-map of $\mathbb{D}$

1. If $C_{\varphi}$ is compact then $\varphi$ does not have an angular derivative at any point in $\partial \mathbb{D}$.

2. If $\varphi$ is univalent and does not have an angular derivative at any point of $\partial \mathbb{D}$, then $C_{\varphi}$ is compact.

Theorem 2.3.5 (The Denjoy-Wolff Theorem). [25, p 75] If $\varphi$ is an analytic self-map of $\mathbb{D}$ with no fixed point in $\mathbb{D}$ then there is a point $w \in \partial \mathbb{D}$ ("the Denjoy-Wolff point of $\varphi$ ") such that the $n$th iterate $\varphi_{n}=\varphi \circ \varphi \circ \ldots \circ \varphi$ ( $n$ times) converges to $w$ uniformly on compact subsets of $\mathbb{D}$.

The following is contained in "The Grand Iteration Theorem".

Theorem 2.3.6. [25, $p$ 78] Suppose $\varphi$ is an analytic self-map of $\mathbb{D}$ that is not an elliptic automorphism.

1. If $\varphi$ has no fixed point in $\mathbb{D}$, then the Denjoy-Wolff point $w \in \partial \mathbb{D}$ of $\varphi$ is a fixed point for $\varphi$ and the angular derivative $\varphi^{\prime}(w)$ exists and satisfies $0<\varphi^{\prime}(w) \leq 1$. 
2. Conversely, if $\varphi$ has a boundary fixed point $w$ at which $\varphi^{\prime}(w) \leq 1$, then $\varphi$ has no fixed point in $\mathbb{D}$, and $w$ is the Denjoy-Wolff point of $\varphi$.

We can conclude the following corollary.

Corollary 2.3.7. [25, $p$ 84] If $C_{\varphi}$ is compact then $\varphi$ has a fixed point in $\mathbb{D}$.

\subsubsection{Adjoint Formula For The Linear Fractional Case}

Let $\varphi=\frac{a z+b}{c z+d}$ be a linear-fractional self-map of $\mathbb{D}$. Then the Krein adjoint of $\varphi$ is defined by

$$
\sigma(z)=\frac{\bar{a} z-\bar{c}}{-\bar{b} z+\bar{d}}
$$

Cowen's adjoint formula (see [11]), relates the adjoint of $C_{\varphi}$ with $C_{\sigma}$ by

$$
C_{\varphi}^{*}=T_{g} C_{\sigma} T_{h}^{*}
$$

where $g(z)=(-\bar{b} z+\bar{d})^{-1}, h(z)=c z+d$ are in $H^{\infty}$. Building on this, in [20] KrieteMacCluer-Moorhouse provide a simple expression for $C_{\varphi}^{*}$ in the Calkin algebra for the following linear fractional case.

Proposition 2.3.8. [20, Theorem 3, Proposition 3] Suppose that $\varphi=\frac{a z+b}{c z+d}$ is a linear-fractional self-map of $\mathbb{D}$, not an automorphism, which satisfies $\varphi(\zeta)=\lambda$ for some $\zeta, \lambda \in \partial \mathbb{D}$. Then

$$
C_{\varphi}^{*} \equiv \frac{1}{\left|\varphi^{\prime}(\zeta)\right|} C_{\sigma} \quad(\bmod \mathcal{K})
$$

where $\sigma$ is the Krein adjoint of $\varphi$. 
In Proposition 5.2.3, we generalize this formula to the bigger class of functions $\mathcal{A}$ defined in Section 4.1.

\subsubsection{Essentially Normal Composition Operators}

Recall that an operator $T$ is normal if it commutes with its adjoint, and essentially normal if $\left[T, T^{*}\right]=T T^{*}-T^{*} T$ is compact. A composition operator $C_{\varphi}$ is normal if and only if $\varphi$ is of the form $\varphi(z)=\alpha z$ with $|\alpha| \leq 1[21, \mathrm{p} 171]$ (result due to Howard Schwartz [21, p 194]). We say that $C_{\varphi}$ is non-trivially essentially normal if it is essentially normal, not compact and not normal.

In [6], Bourdon-Levi-Narayan-Shapiro determined that a composition operator induced by a linear fractional map $\varphi(z)=\frac{a z+b}{c z+d}$ is non-trivially essential normal if and only if $\varphi$ is a parabolic non-automorphism. In particular $\varphi$ has contact with the unit circle $\partial \mathbb{D}$ at one point $\zeta$ and satisfies $\varphi^{\prime}(\zeta)=1$. Additional examples of essentially normal composition operators induced by maps which have order of contact 2 with $\partial \mathbb{D}$ at one point are provided in $[6$, Section 7$]$.

To the best of the author's knowledge, no non-trivially essentially normal composition operators having order of contact $n>2$ with $\partial \mathbb{D}$ were known prior to the present work. 


\subsection{Order of Contact}

We define the notion of order of contact with the boundary both in the context of the unit disk $\mathbb{D}$ and in the context of the upper half-plane $\mathbb{H}$.

Definition 2.1. We say that $\varphi$ has contact with $\partial \mathbb{D}$ at $\zeta$ of order $c>0$ if there is some neighborhood $V$ of $\zeta$ such that $\varphi$ is analytic on $V \cap \mathbb{D}$ and maps $V \cap \mathbb{D}$ into $\mathbb{D}$, and

1. $\varphi(\zeta):=\lim _{z \rightarrow \zeta, z \in \mathbb{D}} \varphi(z)$ exists and $\varphi(\zeta) \in \partial \mathbb{D}$

2. $\frac{1-\left|\varphi\left(e^{i \theta}\right)\right|^{2}}{\left|\varphi(\zeta)-\varphi\left(e^{i \theta}\right)\right|^{c}}$ is essentially bounded above and away from zero as $e^{i \theta} \rightarrow \zeta$.

Definition 2.2. We say that $f$ has contact with $\mathbb{R}$ at 0 of order $c>0$ if there is some neighborhood $W$ of 0 such that $f$ is analytic on $W \cap \mathbb{H}$ and and maps $W \cap \mathbb{H}$ into $\mathbb{H}$, and

1. $f(0):=\lim _{z \rightarrow 0, z \in \mathbb{H}} f(z)$ exists and $f(0) \in \mathbb{R}$

2. $\frac{\operatorname{Im} f(x)}{|f(0)-f(x)|^{c}}$ is essentially bounded above and away from zero as $x \rightarrow 0$ in $\mathbb{R}$.

The following proposition provides some insight and intuition to the definition of order of contact in the upper half-plane by exploring the case where the function is analytic at the point of contact.

Proposition 2.4.1. Suppose that $f$ is analytic in a neighborhood $W$ of 0 with Taylor expansion $f(z)=\sum_{k=0}^{\infty} a_{k} z^{k}$ there, and that $f$ maps 0 into $\mathbb{R}$ and $W \cap \mathbb{H}$ into $\mathbb{H}$. 
Then $f$ maps an interval in $\mathbb{R}$ containing 0 into $\mathbb{R}$ if and only if all the coefficients $a_{k}$ are real. Otherwise, $f$ has order of contact $n=\min \left\{k: \operatorname{Im} a_{k} \neq 0\right\}$ with $\mathbb{R}$ at $0, n$ is even and $\operatorname{Im} a_{n}>0$.

Proof. For all $x \in \mathbb{R}$ we have

$$
\operatorname{Im} f(x)=\sum_{k=0}^{\infty} \operatorname{Im}\left(a_{k}\right) x^{k}
$$

If all the coefficients $a_{k}$ are real then $f$ maps an interval in $\mathbb{R}$ containing 0 into $\mathbb{R}$. Otherwise, let $n$ be the minimal index for which $a_{n}$ is non-real. Then

$$
\operatorname{Im} f(x) \sim \operatorname{Im}\left(a_{n}\right) x^{n}
$$

and so $f$ does not map an interval in $\mathbb{R}$ containing 0 into $\mathbb{R}$. Additionally, we have

$$
|f(0)-f(x)| \sim\left|a_{1}\right| \cdot|x|
$$

and since $f$ maps $W \cap \mathbb{H}$ into $\mathbb{H}$ we have that $a_{1}>0$ and $\operatorname{Im} f(x)>0$ for $x \in \mathbb{R}$ near 0. Thus

$$
0<\frac{\operatorname{Im} f(x)}{|f(0)-f(x)|^{n}} \sim \frac{\operatorname{Im}\left(a_{n}\right) x^{n}}{\left|a_{1}\right|^{n} \cdot|x|^{n}}
$$

and so $n$ is even and $\operatorname{Im}\left(a_{n}\right)>0$. We get that $\frac{\operatorname{Im} f(x)}{|f(0)-f(x)|^{n}} \sim \frac{\operatorname{Im}\left(a_{n}\right)}{\left|a_{1}\right|^{n}}$ and so $f$ has order of contact $n$ with $\mathbb{R}$ at 0 .

We show that the definition in $\mathbb{D}$ corresponds to that in $\mathbb{H}$. To transfer $\mathbb{D}$ to $\mathbb{H}$ we use the family of conformal maps $\tau_{\alpha}: \mathbb{D} \rightarrow \mathbb{H}$ for $\alpha \in \partial \mathbb{D}$, defined by

$$
\tau_{\alpha}(z)=i \frac{\alpha-z}{\alpha+z}
$$


Proposition 2.4.2. Suppose $\varphi$ is analytic at $\zeta$. Then $\varphi$ has order of contact $c$ with $\partial \mathbb{D}$ at $\zeta$ if and only if $f=\tau_{\varphi(\zeta)} \circ \varphi \circ \tau_{\zeta}^{-1}$ has order of contact $c$ with $\mathbb{R}$ at 0 .

Proof. Note that $f$ fixes 0 and that $x=\tau_{\zeta}\left(e^{i \theta}\right)$ tends to 0 in $\mathbb{R}$ if and only if $e^{i \theta}$ tends to $\zeta$ in $\partial \mathbb{D}$. We calculate:

$$
\operatorname{Im} f(x)=\operatorname{Im}\left(\tau_{\varphi(\zeta)}\left(\varphi\left(e^{i \theta}\right)\right)\right)=\operatorname{Im}\left(i \frac{\varphi(\zeta)-\varphi\left(e^{i \theta}\right)}{\varphi(\zeta)+\varphi\left(e^{i \theta}\right)}\right)=\frac{1-\left|\varphi\left(e^{i \theta}\right)\right|^{2}}{\left|\varphi(\zeta)+\varphi\left(e^{i \theta}\right)\right|^{2}}
$$

and

$$
|f(x)|=\left|\tau_{\varphi(\zeta)}\left(\varphi\left(e^{i \theta}\right)\right)\right|=\frac{\left|\varphi(\zeta)-\varphi\left(e^{i \theta}\right)\right|}{\left|\varphi(\zeta)+\varphi\left(e^{i \theta}\right)\right|}
$$

Thus for any $c>0$,

$$
\frac{\operatorname{Im} f(x)}{|f(x)|^{c}}=\frac{1-\left|\varphi\left(e^{i \theta}\right)\right|^{2}}{\left|\varphi(\zeta)-\varphi\left(e^{i \theta}\right)\right|^{c}} \cdot \frac{\left|\varphi(\zeta)+\varphi\left(e^{i \theta}\right)\right|^{c}}{\left|\varphi(\zeta)+\varphi\left(e^{i \theta}\right)\right|^{2}}
$$

Note that

$$
\lim _{e^{i \theta} \rightarrow \zeta} \frac{\left|\varphi(\zeta)+\varphi\left(e^{i \theta}\right)\right|^{c}}{\left|\varphi(\zeta)+\varphi\left(e^{i \theta}\right)\right|^{2}}=\frac{|2 \varphi(\zeta)|^{c}}{|2 \varphi(\zeta)|^{2}}=2^{c-2}
$$

and so $\varphi$ has order of contact $c$ with $\partial \mathbb{D}$ at $\zeta$ if and only if $f$ has order of contact $c$ with $\mathbb{R}$ at 0 .

Order of contact with $\mathbb{D}$ and $\mathbb{H}$ is illustrated in Fig. 2.2, Fig. 2.3 and Fig. 2.4. The maps $f_{1}, f_{2}$ and $f_{3}$ are self-maps of $\mathbb{H}$ constructed using Proposition 3.3.3, with contact with $\mathbb{R}$ at 0 of order 2,4 and 6 respectively and given by

$$
\begin{aligned}
& f_{1}(z)=\frac{-z}{i z-1}=z+i z^{2}+O\left(z^{3}\right) \\
& f_{2}(z)=\frac{i z^{2}-z}{z^{2}+i z-1}=z+z^{3}+i z^{4}+O\left(z^{6}\right) \\
& f_{3}(z)=\frac{z^{3}+i z^{2}-z}{-i z^{3}+2 z^{2}+i z-1}=z+z^{3}+2 z^{5}+i z^{6}+O\left(z^{7}\right) .
\end{aligned}
$$


The maps $\varphi_{1}, \varphi_{2}, \varphi_{3}$ are the corresponding self-maps of $\mathbb{D}$ with contact at 1 , and are given by

$$
\begin{aligned}
\varphi_{1}(z) & =\frac{z+1}{-z+3} \\
\varphi_{2}(z) & =\frac{z^{2}+2 z+1}{z^{2}-2 z+5} \\
\varphi_{3}(z) & =\frac{1}{2} \cdot \frac{z^{3}+z^{2}+z+1}{z^{2}-z+2} .
\end{aligned}
$$

\subsection{Rational Functions}

Rational functions will have a significant role in this thesis. We review some basic definitions and properties and discuss the notion of branches of the inverse function.

Let $r$ be a rational function, and write

$$
r(z)=\frac{p(z)}{q(z)}
$$

where $p$ and $q$ are relatively prime polynomials. Then the degree $d$ of $r$ is defined to be the maximal degree of $p$ and $q$. We think of $r$ as mapping the extended complex

plane $\widehat{\mathbb{C}}=\mathbb{C} \cup\{\infty\}$ into itself. For each point $w \in \widehat{\mathbb{C}}$ the inverse image $r^{-1}(\{w\})$ has $d$ points counting multiplicities [14, Problems 28-32, pages 181-182].

We say $w$ is a regular value of $r$ if $r^{-1}(\{w\})$ consists of $d$ distinct points, and that $w$ is a critical value otherwise. By elementary function theory, $w$ is a critical value for $r$ if and only if $r^{-1}(\{w\})$ contains at least one critical point - a point that has no neighborhood on which $r$ is univalent. For a point $z \in \mathbb{C}, z$ is a critical point if and only if $r^{\prime}(z)=0$. Thus, all but finitely many points of $\hat{\mathbb{C}}$ are regular values for $r$. 
Let $w$ be a regular value of $r$, so that $r^{-1}(\{w\})$ consists of $d$ distinct points $\left\{z_{1}, z_{2}, \ldots, z_{d}\right\}$. Then each point $z_{j}$ is the center of an open disc $\Delta_{j}$ on which $r$ has a univalent restriction. So the restriction of $r$ to $\Delta_{j}$ has an analytic inverse $s_{j}$ on $r\left(\Delta_{j}\right)$. It is called a branch of $r^{-1}$ defined on $r(\Delta)$. We take the intersection of the $r$-images of the discs $\Delta_{1}, \ldots, \Delta_{d}$ to obtain a neighborhood $W$ of $w$ on which all $d$ distinct branches $s_{1}, \ldots, s_{d}$ are defined. By the following proposition, we can extend these $d$ branches to any simply connected domain $V$ containing $W$ and consisting of regular values of $r$.

Proposition 2.5.1. [7, Corollary 3] Suppose $r$ is a rational function and $s$ is a branch of $r^{-1}$ defined on some disc $\Delta$ consisting of regular values of $r$. Suppose $W$ is a simply connected domain consisting of regular values of $r$ and containing $\Delta$. Then $s$ has an analytic continuation to a branch of $r^{-1}$ defined on $W$.

Thus, for any rational function $r$ of degree $d$ and any simply connected domain $V$ consisting of regular values of $r$, there are $d$ distinct branches $s_{1}, \ldots, s_{d}$ of $r^{-1}$ defined on $V$. Furthermore, distinct branches defined on the same connected open set have disjoint images [7, Proposition 1], and so $s_{1}(V), \ldots, s_{d}(V)$ are pairwise disjoint. 
Figure 2.2: Order of contact 2 in $\mathbb{D}$ and $\mathbb{H}$

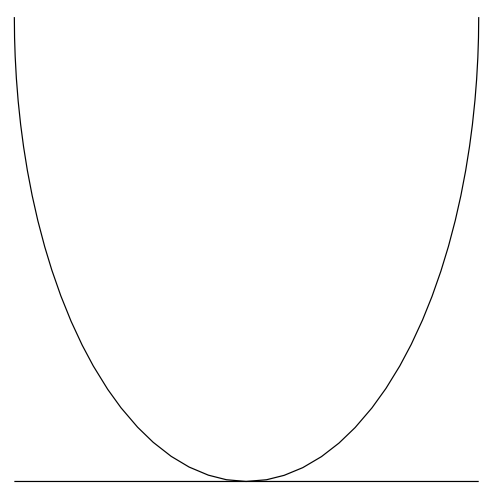

(a) $f_{1}(\mathbb{R})$

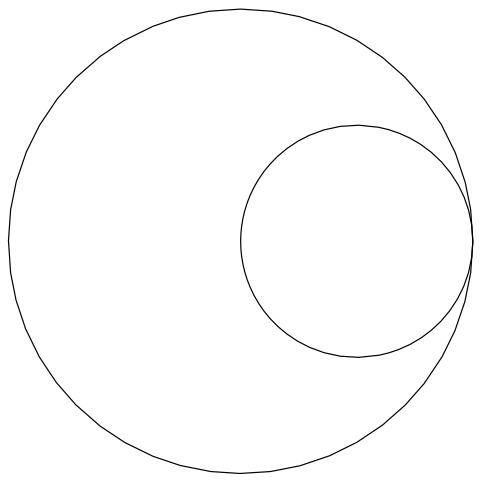

(b) $\varphi_{1}(\partial \mathbb{D})$

Figure 2.3: Order of contact 4 in $\mathbb{D}$ and $\mathbb{H}$

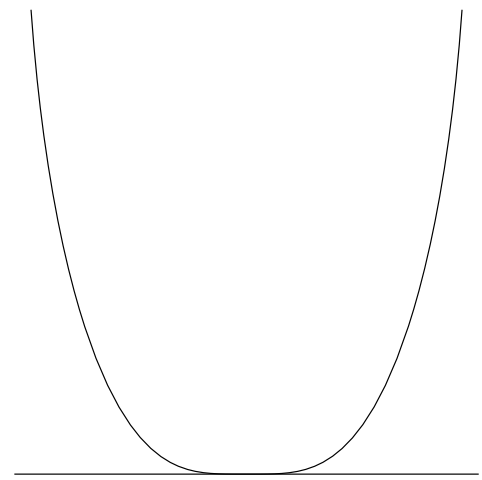

(a) $f_{2}(\mathbb{R})$

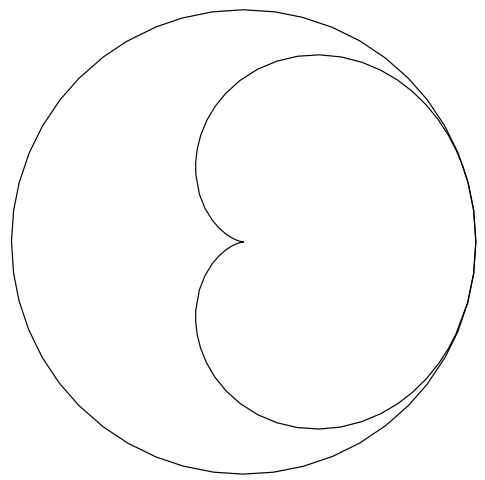

(b) $\varphi_{2}(\partial \mathbb{D})$

Figure 2.4: Order of contact 6 in $\mathbb{D}$ and $\mathbb{H}$

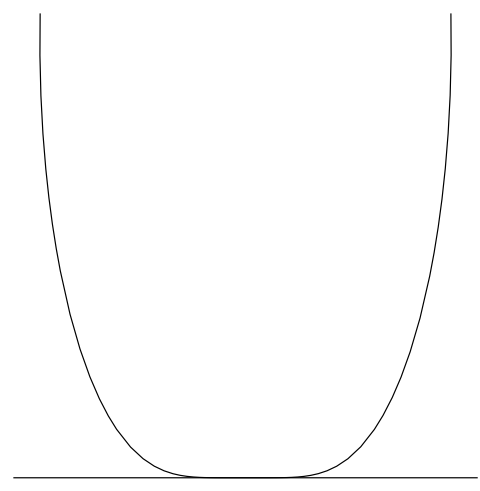

(a) $f_{3}(\mathbb{R})$

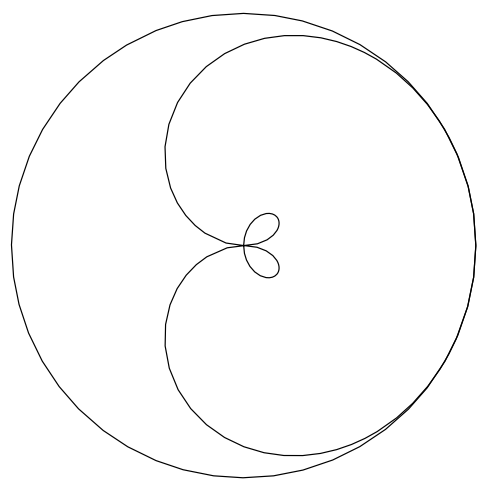

(b) $\varphi_{3}(\partial \mathbb{D})$ 


\section{Chapter 3}

\section{The Boundary Carathéodory-Fejér Problem}

The Carathéodory-Fejér problem $[8,5]$ is to determine whether a given finite sequence of complex numbers comprises the initial Taylor coefficients of an analytic map $f$ mapping the unit disk $\mathbb{D}$ to the upper half-plane $\mathbb{H}$. In [1], Agler-Lykova-Young provide a solvability criterion for the boundary version, denoted $\partial C F \mathcal{P}$, and give a parametrization of all solutions in the real case, denoted $\partial C F \mathcal{P}(\mathbb{R})$.

We review several of the results and techniques used in [1] and the subsequent paper [2], then apply these to obtain a parametrization of all solutions to a slightly different special case of interest - $\partial C F \mathcal{P}(\operatorname{contact}-n)$. We apply this to obtain a parametrization of all analytic self-maps of $\mathbb{H}$ having specified order of contact with $\mathbb{R}$ at 0 , and with varying initial Taylor coefficients. Finally, we construct rational analytic self-maps of the upper half-plane $\mathbb{H}$ that are analytic at 0 and have specified Taylor coefficients there. 


\subsection{The Boundary Carathéodory-Fejér Problem}

In [1], Agler-Lykova-Young study a boundary version of the Carathéodory-Fejér problem, denoted $\partial C F \mathcal{P}$, for functions in the Pick class $\mathcal{P}$ where the interpolation node $x$ lies on the boundary $\mathbb{R}$. Here $\mathcal{P}$ is defined to be the set of analytic functions $f$ mapping the upper half-plane $\mathbb{H}$ into $\mathbb{H} \cup \mathbb{R}$.

Problem 3.1.1 $(\partial C F \mathcal{P})$. Given a point $x \in \mathbb{R}$, a non-negative integer $n$ and numbers $a_{-1}, a_{0}, \ldots, a_{n} \in \mathbb{C}$, determine whether there exists a function $f \in \mathcal{P}$ such that $f$ is analytic in a deleted neighborhood of $x$ and

$$
L_{k}(f, x)=a_{k}, \quad k=-1,0,1, \ldots, n,
$$

where $L_{k}(f, x)$ is the $k$ th Laurent coefficient of $f$ at $x$.

A solvability criterion for this general boundary problem is given in $[1$, Theorem 1.2], which we partially present in Theorem 3.1.3. Parametrization of all solutions for the real case, $\partial C F \mathcal{P}(\mathbb{R})$, where $a_{-1}, a_{0}, \ldots, a_{n} \in \mathbb{R}$, is given in $[1$, Theorem 8.1 , Theorem 8.2, Corollary 8.6].

In the subsequent paper, [2], weaker solutions to $\partial C F \mathcal{P}$, having non-tangential pseudo-Taylor expansions, are considered. For our purposes, unrestricted pseudoTaylor expansions will suffice, and so we will say a function $f \in \mathcal{P}$ is a weak solution of Problem $\partial C F \mathcal{P}$ if $f$ has a pseudo-Taylor expansion

$$
f(z)=a_{0}+a_{1}(z-x)+\ldots+a_{n}(z-x)^{n}+o\left(|z-x|^{n}\right)
$$


as $z \rightarrow 0$ unrestrictedly in $\mathbb{H}$.

Theorem 3.1.2. [2, Theorem 5.2] Problem $\partial C F \mathcal{P}$ with $a_{-1}=0$ has a weak solution if and only if it has a solution that is analytic at $x$.

We are interested in a boundary version of the Carathéodory-Fejér problem where the interpolation node is taken to be $x=0$. We restrict attention to functions $f$ in the Pick class $\mathcal{P}$ which extend analytically to a neighborhood of 0 and denote this subset of the Pick class by $\mathcal{P}_{0}$. Recall from Proposition 2.4.1 that for $f \in \mathcal{P}_{0}, f$ has order of contact $n$ with the real line at 0 if and only if $n$ is even and the initial Taylor coefficient of $f$ at 0 satisfy $a_{0}, a_{1}, \ldots, a_{n-1} \in \mathbb{R}$ and $a_{n} \in \mathbb{H}$. We denote this special case of $\partial C F \mathcal{P}$ where $a_{0}, a_{1}, \ldots, a_{n-1} \in \mathbb{R}$ and $a_{n} \in \mathbb{H}$ by $\partial C F \mathcal{P}($ contact- $n)$. The solvability criterion for $\partial C F \mathcal{P}($ contact- $n)$ case is as follows.

Theorem 3.1.3. [1, Theorem 1.2(2)] Let $n=2 m$ be an even positive integer, $a_{0}, \ldots, a_{n-1} \in \mathbb{R}$ and $a_{n} \in \mathbb{H}$, and let $H_{m}\left(a_{1}, \ldots, a_{n-1}\right)$ be the Hankel matrix defined by

$$
H_{m}\left(a_{1}, \ldots, a_{n-1}\right)=\left[\begin{array}{cccc}
a_{1} & a_{2} & \ldots & a_{m} \\
a_{2} & a_{3} & \ldots & a_{m+1} \\
& & & \\
& . & \ldots & . \\
a_{m} & a_{m+1} & \ldots & a_{n-1}
\end{array}\right]
$$

Then there exists a function $f \in \mathcal{P}_{0}$ with initial Taylor coefficients $a_{0}, \ldots, a_{n}$ at 0 if and only if $H_{m}\left(a_{1}, \ldots, a_{n-1}\right)>0$, i.e., this matrix is positive definite.

Our goal is to derive a parametrization of all the solutions to $\partial C F \mathcal{P}($ contact- $n)$. 
We will use the methods used in [1] for the real case and arrive at a similar parametrization.

\subsection{Reduction and Augmentation}

The main tool we will use is a technique for passing from a function in the Pick class to a simpler one and back again due to G. Julia [18]. We define the reduction and the augmentation (at 0) of a function as follows.

Definition 3.1. For any non-constant function $f \in \mathcal{P}_{0}$ such that $f(0) \in \mathbb{R}$, we define the reduction of $f$ (at 0 ) to be the function $g$ on $\mathbb{H}$ given by the equation

$$
g(z)=-\frac{1}{f(z)-f(0)}+\frac{1}{f^{\prime}(0) z}
$$

Definition 3.2. For any function $g \in \mathcal{P}_{0}$ and any $a_{0} \in \mathbb{R}, a_{1}>0$, we define the augmentation of $g$ (at 0 ) by $a_{0}, a_{1}$ to be the function $f$ on $\mathbb{H}$ given by

$$
f(z)=a_{0}+\frac{1}{\frac{1}{a_{1} z}-g(z)}
$$

The important property of the operations of reduction and augmentation is that they preserve the Pick class.

Proposition 3.2.1. [3, Theorem 3.4]

1. If $f \in \mathcal{P}_{0}$ is non-constant and $f(0) \in \mathbb{R}$, then the reduction of $f$ is in $\mathcal{P}_{0}$ as well.

2. If $g \in \mathcal{P}_{0}$ and $a_{0} \in \mathbb{R}, a_{1}>0$ then the augmentation $f$ of $g$ by $a_{0}, a_{1}$ is in $\mathcal{P}_{0}$ as well and satisfies $f(0)=a_{0}, f^{\prime}(0)=a_{1}$. 
Note that reduction and augmentation are inverse operations on $\mathcal{P}_{0}$, that is,

1. if $f \in \mathcal{P}_{0}$ and if $g$ is the reduction of $f$ then $f$ is the augmentation of $g$ by $f(0), f^{\prime}(0)$;

2. if $g \in \mathcal{P}_{0}$ and if $f$ is the augmentation of $g$ by $a_{0}, a_{1}$ then $g$ is the reduction of $f$.

We will need the following results relating the properties of the Taylor coefficients of a function and those of its reduction.

Proposition 3.2.2. [1, Proposition 2.5] Let $f \in \mathcal{P}_{0}$ satisfy $f^{\prime}(0)>0$, and let $g$ be the reduction of $f$. Let the Taylor expansions of $f$ and $g$ about 0 be

$$
f(z)=\sum_{j=0}^{\infty} a_{j} z^{j}, \quad g(z)=\sum_{j=0}^{\infty} b_{j} z^{j} .
$$

Then the Taylor coefficients $a_{j}$ and $b_{j}$ are related by

$$
\left[\begin{array}{cccc}
a_{1} & 0 & 0 & \ldots \\
a_{2} & a_{1} & 0 & \ldots \\
a_{3} & a_{2} & a_{1} & \ldots \\
\cdot & . & . & \ldots
\end{array}\right]\left[\begin{array}{l}
b_{0} \\
b_{1} \\
b_{2} \\
\cdot
\end{array}\right]=\frac{1}{a_{1}}\left[\begin{array}{l}
a_{2} \\
a_{3} \\
a_{4} \\
\cdot
\end{array}\right]
$$

Corollary 3.2.3. Let $f(z)=\sum_{j=0}^{\infty} a_{j} z^{j}$ and $g(z)=\sum_{j=0}^{\infty} b_{j} z^{j}$ be as in Proposition 3.2.2. Then for any integer $n \geq 2$,

$$
a_{1}, \ldots, a_{n} \text { determine } b_{0}, \ldots, b_{n-2},
$$


and in the other direction

$$
a_{1}, b_{0}, \ldots, b_{n-2} \text { determine } a_{2}, \ldots a_{n}
$$

Furthermore, if $a_{0}, a_{1} \in \mathbb{R}$, then

$$
N=\min \left\{k: \operatorname{Im} a_{k} \neq 0\right\} \quad \text { if and only if } \quad N-2=\min \left\{k: \operatorname{Im} b_{k} \neq 0\right\} .
$$

Proof. For $k=1,2, \ldots$, the $k$ th row of the matrix equation in Proposition 3.2.2 is

$$
a_{k} b_{0}+a_{k-1} b_{1}+\ldots+a_{1} b_{k-1}=\frac{a_{k+1}}{a_{1}} .
$$

Adjusting the index $k$ and solving for the appropriate terms, we get

$$
\begin{array}{ll}
b_{k}=\frac{1}{a_{1}}\left(\frac{a_{k+2}}{a_{1}}-a_{k+1} b_{0}-a_{k} b_{1}-\ldots-a_{2} b_{k-1}\right) & \text { for } k=0,1,2, \ldots \\
a_{k}=a_{1}\left(a_{k-1} b_{0}+a_{k-2} b_{1}+\ldots+a_{1} b_{k-2}\right) & \text { for } k=2,3,4, \ldots
\end{array}
$$

Let $n \geq 2$. Using Eq. (3.2.1) and by induction on $k$, we see that $a_{1}, \ldots, a_{n}$ determine $b_{0}, \ldots, b_{n-2}$. Using Eq. (3.2.2) and again by induction, we see that $a_{1}$ together with $b_{0}, \ldots, b_{n-2}$ determine $a_{2}, \ldots, a_{n}$.

Now suppose that $a_{0}, a_{1}, \ldots, a_{N-1} \in \mathbb{R}$ and $a_{N} \in \mathbb{H}$. Then using Eq. (3.2.1) and by induction on $k=0, \ldots, N-3$ we see that $b_{0}, \ldots b_{N-3} \in \mathbb{R}$. Now, taking imaginary parts of Eq. (3.2.1) for $k=N-2$, we get that $\operatorname{Im}\left(b_{N-2}\right)=\frac{1}{a_{1}^{2}} \operatorname{Im}\left(a_{N}\right)$, so that $b_{N-2} \in \mathbb{H}$. To other direction of the implication follows similarly from Eq. (3.2.2).

Proposition 3.2.4. [1, Corollary 3.3] Let $f(z)=\sum_{j=0}^{j=\infty} a_{j} z^{j}, g(z)=\sum_{j=0}^{j=\infty} b_{j} z^{j}$ be as in Proposition 3.2.2. Then $H_{m}\left(a_{1}, \ldots, a_{2 m-1}\right)>0$ if and only if $H_{m-1}\left(b_{1}, \ldots, b_{2 m-3}\right)>$ 0 . 


\subsection{Parametrization of Solutions to the Contact- $n$}

\section{Case}

We can obtain an alternative expression for the augmentation of a function by introducing the following notation. For a matrix $A=\left[\begin{array}{ll}a_{11} & a_{12} \\ a_{21} & a_{22}\end{array}\right]$, denote the corresponding linear fractional transformation by $L[A]$ :

$$
L[A] h=\frac{a_{11} h+a_{12}}{a_{21} h+a_{22}}
$$

Note that composition of linear fractional transformations corresponds to matrix multiplication, i.e., $L[A] L[B] h=L[A B] h$.

By the definition, the augmentation $f$ of $g$ by $a_{0}, a_{1}$ is

$$
f(z)=a_{0}+\frac{1}{\frac{1}{a_{1} z}-g(z)}=a_{0}-\frac{a_{1} z}{a_{1} z g(z)-1}=\frac{a_{0} a_{1} z g(z)-a_{0}-a_{1} z}{a_{1} z g(z)-1}
$$

and so $f(z)=L\left[A\left(a_{0}, a_{1}\right)(z)\right] g(z)$, where $A\left(a_{0}, a_{1}\right)(z)$ is defined by

$$
A\left(a_{0}, a_{1}\right)(z)=\left[\begin{array}{cc}
a_{0} a_{1} z & -a_{0}-a_{1} z \\
a_{1} z & -1
\end{array}\right] .
$$

Theorem 3.3.1. Let $n=2 m$ be an even positive integer and let $a_{0}, \ldots, a_{n-1} \in \mathbb{R}$ and $a_{n} \in \mathbb{H}$ be such that $H_{m}\left(a_{1}, \ldots, a_{n-1}\right)>0$. Then all solutions to $\partial C F \mathcal{P}($ contact- $n)$ are given by

$$
f(z)=L\left[A\left(a_{0}^{(0)}, a_{1}^{(0)}\right)(z) \cdots A\left(a_{0}^{(m-1)}, a_{1}^{(m-1)}\right)(z)\right] g(z)
$$

for any $g \in \mathcal{P}_{0}$ satisfying $g(0)=a_{0}^{(m)}$, where

$$
a_{0}^{(0)}=a_{0}, a_{0}^{(1)}, \ldots, a_{0}^{(m-1)} \in \mathbb{R}, \quad a_{0}^{(m)} \in \mathbb{H}, \quad a_{1}^{(0)}=a_{1}, a_{1}^{(1)}, \ldots, a_{1}^{(m-1)}>0
$$


are determined by $a_{0}, \ldots, a_{n}$.

Proof. Recall that by Theorem 3.1.3, there exists a solution to $\partial C F \mathcal{P}$ (contact- $n$ ) with initial Taylor coefficients $a_{0}, \ldots a_{n}$, and fix a solution $f_{0}$. We inductively define $f_{k+1} \in$ $\mathcal{P}_{0}$ to be the reduction of $f_{k}$ for $k=1, \ldots, m$, and denote the Taylor coefficients of $f_{k}$ at 0 by $a_{0}^{(k)}, a_{1}^{(k)}, \ldots$ Notice that $f_{k}$ is the augmentation of $f_{k+1}$ by $a_{0}^{(k)}, a_{1}^{(k)}$, so that

$$
f_{k}(z)=L\left[A\left(a_{0}^{(k)}, a_{1}^{(k)}\right)\right] f_{k+1}(z)
$$

and $f_{0}$ can be written as

$$
\begin{aligned}
f_{0}(z) & =L\left[A\left(a_{0}, a_{1}\right)\right] f_{1}(z) \\
& =L\left[A\left(a_{0}^{(0)}, a_{1}^{(0)}\right)(z) A\left(a_{0}^{(1)}, a_{1}^{(1)}\right)(z)\right] f_{2}(z)=\cdots \\
& =L\left[A\left(a_{0}^{(0)}, a_{1}^{(0)}\right)(z) \cdots A\left(a_{0}^{(m-1)}, a_{1}^{(m-1)}\right)(z)\right] f_{m}(z) .
\end{aligned}
$$

By Corollary 3.2.3, all the Taylor coefficients listed below are determined by $a_{0}, \ldots, a_{n}$ and do not depend on our choice of $f_{0}$.

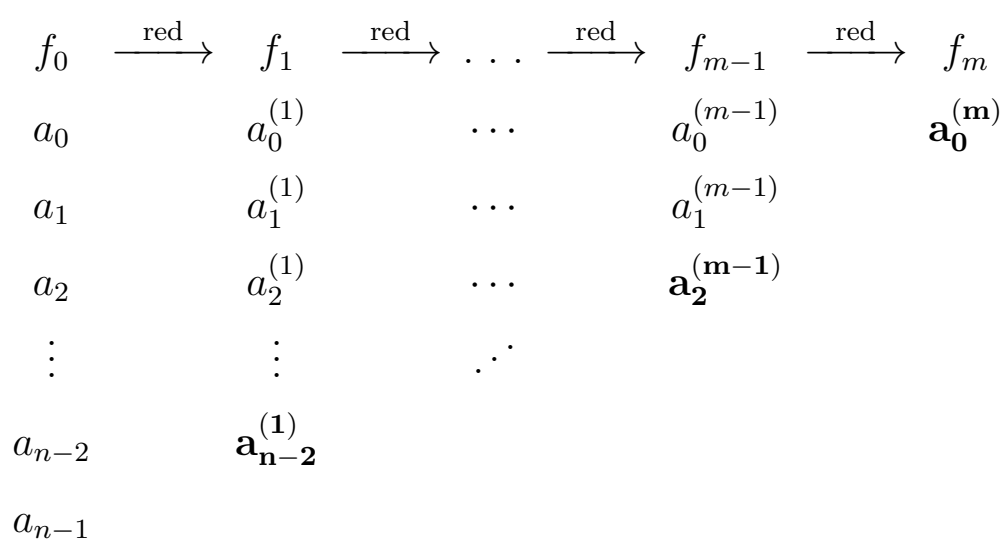

$a_{n}$

In general, for $k=1, \ldots, m$ we have that $a_{0}^{(k)}, \ldots a_{n-2 k}^{(k)}$ are determined. Corollary 3.2.3 further shows that $a_{0}^{(k)}, \ldots a_{n-2 k-1}^{(k)} \in \mathbb{R}$ and $a_{n-2 k}^{(k)} \in \mathbb{H}$, i.e., all the non-bold coefficients 
above are in $\mathbb{R}$ and all the bold coefficient are in $\mathbb{H}$. In particular, we get that

$$
a_{0}^{(0)}=a_{0}, a_{0}^{(1)}, \ldots, a_{0}^{(m-1)} \in \mathbb{R}, \quad a_{0}^{(m)} \in \mathbb{H} .
$$

Additionally, by Proposition 3.2.4, for $k=1, \ldots, m-1$ the Hankel matrix $H_{m-k}\left(a_{1}^{(k)}, \ldots, a_{n-2 k-1}^{(k)}\right)$ is positive and so in particular we get that

$$
a_{1}^{(0)}=a_{1}, a_{1}^{(1)}, \ldots, a_{1}^{(m-1)}>0
$$

Note that since $f_{0}, \ldots, f_{m-1}$ are all real valued at 0 and non-constant, taking the above reductions makes sense.

Now let $F_{0}$ be any solution of $\partial C F \mathcal{P}($ contact- $n)$ with $a_{0}, \ldots, a_{n}$, and let $F_{k}$ denote the $k$ th reduction of $F_{0}$. As discussed above, the first $n-2 k+1$ Taylor coefficients of $F_{k}$ are $a_{0}^{(k)}, \ldots a_{n-2 k}^{(k)}$, and so $F_{0}$ can be written as

$$
F_{0}(z)=L\left[A\left(a_{0}^{(0)}, a_{1}^{(0)}\right)(z) \cdots A\left(a_{0}^{(m-1)}, a_{1}^{(m-1)}\right)(z)\right] F_{m}(z)
$$

where $F_{m}$ satisfies $F_{m}(0)=a_{0}^{(m)}$.

Conversely, for any $F_{m} \in \mathcal{P}_{0}$ satisfying $F_{m}(0)=a_{0}^{(m)}$ we inductively define $F_{k}$ for $k=m-1, \ldots, 0$ to be the augmentation of $F_{k+1}$ by $a_{0}^{(k)}, a_{1}^{(k)}$, i.e.,

$$
F_{k}(z)=L\left[A\left(a_{0}^{(k)}, a_{1}^{(k)}\right)\right] F_{k+1}(z)
$$

By the second assertion in Corollary 3.2.3, the first $n-2 k+1$ Taylor coefficients of $F_{k}$ are again $a_{0}^{(k)}, \ldots a_{n-2 k}^{(k)}$, and so the function

$$
F_{0}(z)=L\left[A\left(a_{0}^{(0)}, a_{1}^{(0)}\right)(z) \cdots A\left(a_{0}^{(m-1)}, a_{1}^{(m-1)}\right)(z)\right] F_{m}(z)
$$

is indeed a solution to $\partial C F \mathcal{P}($ contact- $n)$ with $a_{0}, \ldots, a_{n}$. 
Theorem 3.3.2. Let $n=2 m$ be an even positive integer. Let $a_{0}, \ldots, a_{n-1} \in \mathbb{R}$ and $a_{n} \in \mathbb{H}$ be such that $H_{m}\left(a_{1}, \ldots, a_{n-1}\right)>0$. Then all solutions for $\partial C F \mathcal{P}($ contact- $n)$ are parametrized by

$$
f(z)=\frac{p(z) h(z)+q(z)}{r(z) h(z)+s(z)}
$$

where $p, q, r, s$ are polynomials with real coefficients of degree at most $m$ determined by $a_{0}, \ldots, a_{n}$ and satisfying for some $K>0$,

$$
(p s-q r)(z)=K z^{n}
$$

and $h \in \mathcal{P}_{0}$ satisfies $h(0)=h_{0}$, where $h_{0}$ is determined by $a_{0}, \ldots, a_{n}$.

Proof. Let $a_{0}^{(0)}, \ldots, a_{0}^{(m)}$ and $a_{1}^{(0)}, \ldots, a_{1}^{(m-1)}$ be as in Theorem 3.3.1, so that all solutions are parametrized by

$$
f(z)=L\left[A\left(a_{0}^{(0)}, a_{1}^{(0)}\right)(z) \cdots A\left(a_{0}^{(m-1)}, a_{1}^{(m-1)}\right)(z)\right] h(z)
$$

where $h$ is any function in $\mathcal{P}_{0}$ with $h(0)=a_{0}^{(m)}$.

We define the polynomials $p, q, r, s$ by

$$
\left[\begin{array}{cc}
p(z) & q(z) \\
r(z) & s(z)
\end{array}\right]=A\left(a_{0}^{(0)}, a_{1}^{(0)}\right)(z) \cdots A\left(a_{0}^{(m-1)}, a_{1}^{(m-1)}\right)(z)
$$

so that

$$
f(z)=L\left[\begin{array}{ll}
p(z) & q(z) \\
r(z) & s(z)
\end{array}\right] h(z)=\frac{p(z) h(z)+q(z)}{r(z) h(z)+s(z)} .
$$

Recall that

$$
A\left(\alpha_{0}, \alpha_{1}\right)(z)=\left[\begin{array}{cc}
\alpha_{0} \alpha_{1} z & -\alpha_{0}-\alpha_{1} z \\
\alpha_{1} z & -1
\end{array}\right]
$$


and so $p, q, r, s$ are real polynomials of degree at most $m$. Notice that

$$
\operatorname{det} A\left(\alpha_{0}, \alpha_{1}\right)(z)=-\alpha_{0} \alpha_{1} z+\alpha_{0} \alpha_{1} z+\alpha_{1}^{2} z^{2}=\alpha_{1}^{2} z^{2}
$$

so by taking determinants we get that

$$
(p s-q r)(z)=\left(a_{1}^{(0)}\right)^{2} z^{2} \cdots\left(a_{1}^{(m-1)}\right)^{2} z^{2}=K z^{n} .
$$

As a corollary to Theorem 3.3.1, we can now provide a parametrization of all contact $n$ functions.

Proposition 3.3.3. All functions $f \in \mathcal{P}_{0}$ with order of contact $n=2 m$ with $\mathbb{R}$ at 0 are parametrized by

$$
f(z)=L\left[A\left(a_{0}^{(0)}, a_{1}^{(0)}\right)(z) \cdots A\left(a_{0}^{(m-1)}, a_{1}^{(m-1)}\right)(z)\right] g(z)
$$

where $g \in \mathcal{P}_{0}$ satisfies $g(0) \in \mathbb{H}$ and

$$
a_{0}^{(0)}, a_{0}^{(1)}, \ldots, a_{0}^{(m-1)} \in \mathbb{R}, \quad a_{1}^{(0)}, a_{1}^{(1)}, \ldots, a_{1}^{(m-1)}>0 .
$$

Furthermore, for any $f \in \mathcal{P}_{0}$, these parameters are uniquely determined.

Proof. Let $f \in \mathcal{P}_{0}$ have order of contact $n=2 m$ with $\mathbb{R}$ at 0 with initial Taylor coefficients $a_{0}, \ldots, a_{n}$. Then by Theorem $3.1 .3, H_{m}\left(a_{1}, \ldots, a_{n-1}\right)>0$ and so by Theorem 3.3.1 $f$ is of the desired form.

For the other direction note that any $f$ of this form is obtained by applying $m$ augmentations to $g \in \mathcal{P}_{0}$, and so $f \in \mathcal{P}_{0}$. Recall that by Corollary 3.2.3, augmentation 
increases the order of contact with $\mathbb{R}$ by 2 , and so $g(0) \in \mathbb{H}$ implies that $f$ has order of contact $2 m=n$.

To see uniqueness, notice that if

$$
f(z)=L\left[A\left(a_{0}^{(0)}, a_{1}^{(0)}\right)(z) \cdots A\left(a_{0}^{(m-1)}, a_{1}^{(m-1)}\right)(z)\right] g(z)
$$

then $a_{0}^{(k)}=f_{k}(0), a_{1}^{(k)}=f_{k}^{\prime}(0)$ and $g=f_{m}$ where $f_{k}$ denotes the $k$ th reduction of $f$.

\subsection{Rational Functions with Specified Taylor Co- efficients}

We turn our attention to construction of simple solutions to $\partial C F \mathcal{P}$. Initially we construct rational maps $f \in \mathcal{P}_{0}$ which map 0 into $\mathbb{R}$ and the rest of $\widehat{\mathbb{R}}=\mathbb{R} \cup\{\infty\}$ into $\mathbb{H}$ and have specified initial Taylor coefficients at 0 . We then add the condition that 0 is a regular value for $f$, i.e., $f^{-1}(\{0\})$ consists of $d$ distinct points where $d$ is the degree of $f$. We begin by showing that augmentation preserves the properties we are interested in.

Proposition 3.4.1. Let $g \in \mathcal{P}_{0}$ be a rational function of degree $d$. Then any augmentation $f$ of $g$ is a rational function of degree $d+1$.

Proof. Let $g=\frac{p(z)}{q(z)} \in \mathcal{P}_{0}$ be a rational function of degree $d$, with $p(z)$ and $q(z)$ are relatively prime, and let $f \in \mathcal{P}_{0}$ be the augmentation of $g$ by $a_{0}, a_{1}$. Then by the 
definition of augmentation,

$$
f(z)=a_{0}+\frac{1}{\frac{1}{a_{1} z}-\frac{p(z)}{q(z)}},
$$

and so it suffices to show that the rational function

$$
\frac{1}{a_{1} z}-\frac{p(z)}{q(z)}=\frac{q(z)-a_{1} z p(z)}{a_{1} z q(z)}=\frac{r(z)}{s(z)}
$$

has degree $d+1$. We see that $\max \{\operatorname{deg} r, \operatorname{deg} s\}=d+1$, and it remains to show that $r(z)$ and $s(z)$ are relatively prime.

First note that $q(z)$ is not divisible by $z$ since $g$ is analytic at 0 , and so $z$ is not a common divisor of $r(z)$ and $s(z)$. Now assume that $z-\alpha$ is a common divisor of $r(z)$ and $s(z)$ for some $\alpha \neq 0$. Then $z-\alpha$ divides $a_{1} z q(z)$ implies $z-\alpha$ divides $q(z)$, and $z-\alpha$ divides both $q(z)$ and $q(z)-a_{1} z p(z)$ implies $z-\alpha$ divides $p(z)$. This contradicts the assumption that $p(z)$ and $q(z)$ are relatively prime, and so $r(z)$ and $s(z)$ are indeed relatively prime.

Proposition 3.4.2. Let $g \in \mathcal{P}_{0}$ mapping $\widehat{\mathbb{R}} \backslash\{0\}$ into $\mathbb{H}$. Let $f=L\left[A\left(a_{0}, a_{1}\right)\right] g$ with $\operatorname{Im} a_{0} \geq 0$ and $a_{1}>0$ (note that for $a_{0}$ real, $f$ is an augmentation of $g$ ). Then $f$ also maps $\widehat{\mathbb{R}} \backslash\{0\}$ into $\mathbb{H}$.

Proof. By the definition of $A\left(a_{0}, a_{1}\right)$,

$$
f(z)=\frac{a_{0} a_{1} z g(z)-a_{0}-a_{1} z}{a_{1} z g(z)-1}=a_{0}+\frac{1}{\frac{1}{a_{1} z}-g(z)} .
$$

Let $x \in \widehat{\mathbb{R}} \backslash\{0\}$, so that $g(x) \in \mathbb{H}$. Then

$$
\operatorname{Im}\left(\frac{1}{a_{1} x}-g(x)\right)=-\operatorname{Im} g(x)<0
$$


and so

$$
\operatorname{Im} f(x)=\operatorname{Im} a_{0}+\operatorname{Im}\left(\frac{1}{\frac{1}{a_{1} x}-g(x)}\right)>0 .
$$

Proposition 3.4.3. Let $f$ be in $\mathcal{P}$ and suppose that for some $n=2 m$, $f$ has pseudo Taylor coefficients

$$
\lim _{z \rightarrow 0} \frac{f^{(k)}(z)}{k !}=a_{k} \quad \text { for } k=0,1, \ldots, n
$$

where the limits are taken unrestrictedly in $\mathbb{H}$, such that $a_{0}, a_{1}, \ldots, a_{n-1} \in \mathbb{R}$ and $a_{n} \in \mathbb{H}$.

Then there exists a degree $m$ rational function $F \in \mathcal{P}_{0}$ that maps $\widehat{\mathbb{R}} \backslash\{0\}$ into $\mathbb{H}$ and has initial Taylor coefficients $a_{0}, \ldots, a_{n}$ at $z=0$.

Proof. By Theorem 3.1.2 and Theorem 3.1.3, $H_{m}\left(a_{1}, \ldots, a_{n-1}\right)>0$ and so by Theorem 3.3.1 with $g(z) \equiv a_{0}^{(m)}$, we have that

$$
F(z)=L\left[A\left(a_{0}^{(0)}, a_{1}^{(0)}\right)(z) \cdots A\left(a_{0}^{(m-1)}, a_{1}^{(m-1)}\right)(z)\right] a_{0}^{(m)}
$$

is in $\mathcal{P}_{0}$ with the desired Taylor coefficients. Here $a_{0}^{(0)}=a_{0}, a_{0}^{(1)}, \ldots, a_{0}^{(m-1)} \in \mathbb{R}$, $a_{0}^{(m)} \in \mathbb{H}$ and $a_{1}^{(0)}=a_{1}, a_{1}^{(1)}, \ldots, a_{1}^{(m-1)}>0$ are determined by $a_{0}, \ldots, a_{n}$.

Note that $F$ is obtained by applying $m$ augmentations to $g(z) \equiv a_{0}^{(m)}$. Thus by Proposition 3.4.1, $F$ is a degree $m$ rational function, and by Proposition 3.4.2 applied $m$ times, $F$ maps $\widehat{\mathbb{R}} \backslash\{0\}$ into $\mathbb{H}$. 
Proposition 3.4.4. Let $f$ be in $\mathcal{P}$ and suppose that for some $n=2 m$, $f$ has pseudo Taylor coefficients

$$
\lim _{z \rightarrow 0} \frac{f^{(k)}(z)}{k !}=a_{k} \quad \text { for } k=0,1, \ldots, n
$$

where the limits are taken unrestrictedly in $\mathbb{H}$, such that $a_{0}, a_{1}, \ldots, a_{n-1} \in \mathbb{R}$ and $a_{n} \in \mathbb{H}$.

Then there exists a degree $m+1$ rational function $f_{0} \in \mathcal{P}_{0}$ that has 0 as a regular value, maps $\widehat{\mathbb{R}} \backslash\{0\}$ into $\mathbb{H}$ and has initial Taylor coefficients $a_{0}, \ldots, a_{n}$ at $z=0$.

Proof. By Theorem 3.1.2 and Theorem 3.1.3, $H_{m}\left(a_{1}, \ldots, a_{n-1}\right)>0$ and so by Theorem 3.3.1 we have that for any $g \in \mathcal{P}_{0}$ with $g(0)=a_{0}^{(m)}$,

$$
F(z)=L\left[A\left(a_{0}^{(0)}, a_{1}^{(0)}\right)(z) \cdots A\left(a_{0}^{(m-1)}, a_{1}^{(m-1)}\right)(z)\right] g(z)
$$

is in $\mathcal{P}_{0}$ with the desired Taylor coefficients. Here $a_{0}^{(0)}=a_{0}, a_{0}^{(1)}, \ldots, a_{0}^{(m-1)} \in \mathbb{R}$, $a_{0}^{(m)} \in \mathbb{H}$ and $a_{1}^{(0)}=a_{1}, a_{1}^{(1)}, \ldots, a_{1}^{(m-1)}>0$ are determined by $a_{0}, \ldots, a_{n}$.

For any $w \in \mathbb{H}$, we define $g_{w}(z)=L\left[A\left(a_{0}^{(m)}, 1\right)(z)\right] w=a_{0}^{(m)}+\frac{z}{1-w z}$. Then $g$ is a degree 1 rational function in $\mathcal{P}_{0}$ and by Proposition 3.4.2, $g_{w}$ maps $\widehat{\mathbb{R}}$ into $\mathbb{H}$.

Let $F_{w}$ denote the function $F$ above resulting from the choice $g=g_{w}$ and note that $F_{w}$ is obtained by applying $m$ augmentations to $g_{w}$. Thus by Proposition 3.4.1, $F_{w}$ is a degree $m+1$ rational function, and by Proposition 3.4.2 applied $m$ times, $F_{w}$ maps $\widehat{\mathbb{R}} \backslash\{0\}$ into $\mathbb{H}$.

It remains to find a $w \in \mathbb{H}$ such that 0 is a regular value for $F_{w}$, i.e., $F_{w}(z)=0$ 
has $m+1$ distinct solutions. Let $p, q, r, s$ be defined by

$$
\left[\begin{array}{cc}
p(z) & q(z) \\
r(z) & s(z)
\end{array}\right]=A\left(a_{0}^{(0)}, a_{1}^{(0)}\right)(z) \cdots A\left(a_{0}^{(m-1)}, a_{1}^{(m-1)}\right)(z) A\left(a_{0}^{(m)}, 1\right)(z),
$$

so that $p, q, r, s$ are polynomials of degree at most $m+1$, and

$$
F_{w}(z)=\frac{p(z) w+q(z)}{r(z) w+s(z)}
$$

Since the degree of $F_{w}$ is $m+1, p(z) w+q(z)$ and $r(z) w+s(z)$ have no common roots and so it suffices to find $w$ such that $p(z) w+q(z)=0$ has $m+1$ distinct roots. Note that $p$ and $q$ have no non-zero roots in common, since by taking determinants, we have that

$$
(p s-q r)(z)=\left(a_{1}^{(0)}\right)^{2} z^{2} \cdots\left(a_{1}^{(m)}\right)^{2} z^{2}=K z^{n+2}, \quad K \neq 0 .
$$

This equation also shows that at least one of $p$ and $q$ has degree $m+1$. Suppose for the moment that $F_{w}(0)=a_{0} \neq 0$, i.e $z=0$ is not a common root for $p$ and $q$. Then the function $-\frac{q(z)}{p(z)}$ is a rational function of degree $m+1$ and so all but finitely many $w \in \mathbb{C}$ are regular values. For any choice $w \in \mathbb{H}$ that is a regular value of $-\frac{q(z)}{p(z)}, F_{w}$ is the desired function.

If $F_{w}(0)=a_{0}=0$ then $z=0$ is a common root for $p$ and $q$, however $F_{w}^{\prime}(0)=a_{1} \neq 0$ and so $z=0$ is a solution of multiplicity one to $p(z) w+q(z)=0$. Thus the function $-\frac{q(z)}{p(z)}$ is a rational function of degree $m$, and so we can find $w \in \mathbb{H}$ with pre-image consisting of $m$ points. These $m$ points and the point $z=0$ are then the $m+1$ distinct solutions to $F_{w}(z)=0$. 


\section{Chapter 4}

\section{Relations In The Calkin Algebra}

In [19], Kriete-Moorhouse investigate compactness of linear combinations of composition operators where the inducing maps have certain properties of analyticity. We review some definitions and results from [19], then apply our results from Chapter 3 to obtain a decomposition of a composition operator in the Calkin algebra into a sum of composition operators induced by rational or basic functions (see Definition 4.1).

Additionally, we review a result from [19] regarding weighted composition operators in the Calkin algebra and use this result in the proof of a similar result concerning weighted adjoints of composition operators.

\subsection{The Class of Functions $\mathcal{A}$}

We will work in the class of functions $\mathcal{A}$ (denoted $\mathcal{S}$ in [19]) consisting of analytic self-maps $\varphi$ of $\mathbb{D}$ with certain properties of analyticity. The model for a function in $\mathcal{A}$ is an analytic self-map of $\mathbb{D}$ which extends analytically to a neighborhood of $\mathbb{D}$ and is not a Blaschke product. In particular, we restrict the number of points of contact 
with the unit circle, and require relatively nice behavior at the points of contact. To make a precise definition, we first discuss Clark measures.

\subsubsection{Clark Measures}

Let $\varphi$ be an analytic self-map of $\mathbb{D}$. If $|\alpha|=1$, there exists a finite positive Borel measure $\mu_{\alpha}$ on $\partial \mathbb{D}$ such that

$$
\frac{1-|\varphi(z)|^{2}}{|\alpha-\varphi(z)|^{2}}=\operatorname{Re}\left(\frac{\alpha+\varphi(z)}{\alpha-\varphi(z)}\right)=\int_{\partial \mathbb{D}} P_{z}\left(e^{i t}\right) d \mu_{\alpha}(t)
$$

for $z$ in $\mathbb{D}$, where

$$
P_{z}\left(e^{i t}\right)=\frac{1-|z|^{2}}{\left|e^{i t}-z\right|^{2}}
$$

is the Poisson kernel at $z$. The existence of $\mu_{\alpha}$ follows since the left side the equation above is a positive harmonic function. The measures $\mu_{\alpha}$ are called the Clark measures of $\varphi$. More information about Clark measures can be found in [9] and Sarason [24].

The singular part of the measure, $\mu_{\alpha}^{s}$, is carried by $\varphi^{-1}(\{\alpha\})$, the set of those $\zeta \in \partial \mathbb{D}$ where $\varphi(\zeta)$, the non-tangential limit of $\varphi$ at $\zeta$, exists and equals $\alpha$. The measure $\mu_{\alpha}^{s}$ is the sum of the pure point measure

$$
\mu_{\alpha}^{p p}=\sum_{\varphi(\zeta)=\alpha} \frac{1}{\left|\varphi^{\prime}(\zeta)\right|} \delta_{\zeta}
$$

where $\delta_{\zeta}$ is the unit point mass at $\zeta$, and a continuous singular measure $\mu_{\alpha}^{c s}$.

We write

$$
E(\varphi)=\overline{\bigcup_{|\alpha|=1} \operatorname{spt}\left(\mu_{\alpha}^{s}\right)}
$$


where $\operatorname{spt}(\mu)$ denotes the closed support of a measure $\mu$. Note that for any $\varphi$,

$$
F(\varphi)=\{\zeta: \varphi \text { has finite angular derivative at } \zeta\}
$$

is a subset of $E(\varphi)$. If $E(\varphi)$ is a finite set then the continuous singular measure $\mu_{\alpha}^{c s}$ all vanish, and we get that $E(\varphi)=F(\varphi)$.

\subsubsection{Definition of $\mathcal{A}$}

We define the class $\mathcal{A}$ to be the set of analytic self-maps $\varphi$ of $\mathbb{D}$ with $E(\varphi)=F(\varphi)$ and such that for each point $\zeta \in F(\varphi)$, there exist an $n$ such that $\varphi$ has order of contact $n$ at $\zeta$ and complex numbers $a_{0}, a_{1}, \ldots, a_{n}$ such that

$$
\varphi(z)=a_{0}+a_{1}(z-\zeta)+\ldots+a_{n}(z-\zeta)^{n}+o\left(|z-\zeta|^{n}\right)
$$

as $z \rightarrow \zeta$ unrestrictedly in $\mathbb{D}$.

For $\varphi \in \mathcal{A}$ with order of contact $n$ at $\zeta$, we define the derivatives of $\varphi$ at $\zeta$ by

$$
\varphi^{(j)}(\zeta):=\angle \lim _{z \rightarrow \zeta} \varphi^{(j)}(z)=j ! a_{j}
$$

for $j=1, \ldots, n$ and note that these limits do exist (see, the argument in [24, $\mathrm{p} 47]$ ).

Proposition 4.1.1. $\mathcal{A}$ contains all self-maps of $\mathbb{D}$ that extend analytically to a neighborhood of $\mathbb{D}$ that are not finite Blaschke products.

Proof. Let $\varphi$ be a self-map of $\mathbb{D}$ which extends analytically to $V \supset \mathbb{D}$ and is not a finite Blaschke product. Let $S=\{\zeta \in \partial \mathbb{D}:|p(\zeta)|=1\}$ be the set of points where $\varphi$ 
has contact with $\partial \mathbb{D}$. Then $S$ consists of zeros of the analytic function

$$
f(z):=\varphi(z)-\varphi_{e}(z)
$$

where $\varphi_{e}=\rho \circ \varphi \circ \rho$, and $\rho$ is the inversion in the unit circle defined by $\rho(z):=1 / \bar{z}$. If $S$ is infinite then the set of zeros of $f$ has an accumulation point in $V$ and so $f$ is identically 0 on $V$. In this case $\varphi ( e ^ { i \theta } ) = 1 \longdiv { \varphi ( e ^ { i \theta } ) }$ for all $e^{i \theta}$, i.e $\varphi$ is inner. But since $\varphi$ is an inner function analytic in a neighborhood of $\mathbb{D}, \varphi$ must be a finite Blaschke product, contradicting our assumption. Thus $S$ is finite and so $E(\varphi)$ is finite. Since $\varphi$ is analytic at each point $\zeta \in F(\varphi)=E(\varphi)$ and maps a small arc containing $\zeta$ onto a curve with contact with $\partial \mathbb{D}$ at exactly one point, $\varphi$ has finite order of contact at $\zeta$. Lastly, for $n$ equal to the order of contact of $\varphi$ with $\partial \mathbb{D}$ at $\zeta$, we can write

$$
\varphi(z)=a_{0}+a_{1}(z-\zeta)+\ldots+a_{n}(z-\zeta)^{n}+o\left(|z-\zeta|^{n}\right)
$$

using the Taylor coefficients of $\varphi$ at $\zeta$. Thus, $\varphi$ is indeed in $\mathcal{A}$.

\subsection{Linear Relations for Composition Operators in}

\section{the Calkin Algebra}

In [19], Kriete-Moorhouse show that information relating to compactness of a linear combination of compositions operators

$$
c_{1} C_{\varphi_{1}}+\ldots+c_{r} C_{\varphi_{r}}
$$


where $\varphi_{1}, \ldots, \varphi_{r} \in \mathcal{A}$, is carried by the behavior of the functions $\varphi_{j}$ at their points of contact with the unit circle. More precisely, the relevant information for $\varphi$ at a point of contact $\zeta$ is

$$
D_{n}(\varphi, \zeta)=\left(\varphi(\zeta), \varphi^{\prime}(\zeta) \ldots, \varphi^{(n)}(\zeta)\right)
$$

where $n$ is the order of contact of $\varphi$ with the unit circle at $\zeta$.

The following result determines compactness of a linear combination of composition operators for operators induced by functions in $\mathcal{A}$.

Theorem 4.2.1. [19, Theorem 5.13] Let $\varphi_{1}, \ldots, \varphi_{r}$ in $\mathcal{A}$ and write $F$ for the union $F\left(\varphi_{1}\right) \cup \ldots \cup F\left(\varphi_{r}\right)$, a finite set. For $\zeta$ in $F$ and $k=2,4,6, \ldots$, let

$$
\mathbb{N}_{k}(\zeta)=\left\{j: F\left(\varphi_{j}\right) \text { contains } \zeta \text { and } k \text { is the order of contact of } \varphi_{j} \text { at } \zeta\right\}
$$

and let

$$
\mathcal{E}_{k}(\zeta)=\left\{D_{k}\left(\varphi_{j}, \zeta\right): j \text { is in } \mathbb{N}_{k}(\zeta)\right\}
$$

Given complex numbers $c_{1}, \ldots, c_{r}$, the following are equivalent:

1. $c_{1} C_{\varphi_{1}}++c_{n} C_{\varphi_{r}}$ is compact

2. for every $\zeta$ in $F$, every even $k \geq 2$ and every $\boldsymbol{d}$ in $\mathcal{E}_{k}(\zeta)$,

$$
\sum_{\substack{j \in \mathbb{N}_{k}(\zeta) \\ D_{k}\left(\varphi_{j}, \zeta\right)=d}} c_{j}=0
$$

Our goal is to decompose $C_{\varphi}$, modulo the ideal $\mathcal{K}$ of compact operators, into a sum of composition operators induced by rational functions, each having contact with the unit circle at exactly one point. We begin by proving the following lemma. 
Lemma 4.2.2. Let $\varphi \in \mathcal{A}$ and $\zeta \in F(\varphi)$. Let $n=2 m$ denote the order of contact of $\varphi$ with $\partial \mathbb{D}$ at $\zeta$. Then there exists a degree $m$ rational function $\varphi_{0}$ with order of contact $n$ with $\partial \mathbb{D}$ at $\zeta$ and mapping the rest of $\overline{\mathbb{D}}$ into $\mathbb{D}$ which satisfies $D_{n}\left(\varphi_{0}, \zeta\right)=D_{n}(\varphi, \zeta)$.

Proof. Let $\lambda=\varphi(\zeta)$ and define

$$
f=\tau_{\lambda} \circ \varphi \circ \tau_{\zeta}^{-1}
$$

where $\tau_{\alpha}(z)=i \frac{\alpha-z}{\alpha+z}$. Then $f \in \mathcal{P}$ fixes 0 and since $\varphi \in \mathcal{A}, f$ has pseudo Taylor coefficients

$$
\lim _{z \rightarrow 0} \frac{f^{(k)}(z)}{k !}=a_{k} \quad \text { for } k=0,1, \ldots, n
$$

such that $a_{0}, a_{1}, \ldots, a_{n-1} \in \mathbb{R}$ and $a_{n} \in \mathbb{H}$.

Thus, by Proposition 3.4.3, there exists a degree $m$ rational function $F \in \mathcal{P}_{0}$ that maps $\widehat{\mathbb{R}} \backslash\{0\}$ into $\mathbb{H}$ and has initial Taylor coefficients $a_{0}, \ldots, a_{n}$ at $z=0$. We let $\varphi_{0}$ be the self-map of $\mathbb{D}$ defined by

$$
\varphi_{0}=\tau_{\lambda}^{-1} \circ F \circ \tau_{\zeta}
$$

Note that $\tau_{\alpha}$ preserves the degree of a rational function, and so $\varphi_{0}$ is a degree $m$ rational function. As a consequence of the boundary behavior of $F, \varphi_{0}$ maps $\zeta$ to $\lambda$ and the rest of $\overline{\mathbb{D}}$ into $\mathbb{D}$. Since $D_{n}\left(\varphi_{0}, \zeta\right)$ is determined by $D_{n}\left(\tau_{\zeta}, \zeta\right), D_{n}(F, 0)$ and $D_{n}\left(\tau_{\lambda}^{-1}, 0\right)$ (see Theorem 5.3.1), $\varphi_{0}$ satisfies $D_{n}\left(\varphi_{0}, \zeta\right)=D_{n}(\varphi, \zeta)$. Lastly, since $F$ has order of contact $n$ with $\mathbb{R}$ at 0 and by Proposition $2.4 .2, \varphi_{0}$ has order of contact $n$ with $\partial \mathbb{D}$ at $\zeta$. 
We combine this result with Theorem 4.2.1 to obtain our first decomposition theorem.

Theorem 4.2.3. Let $\varphi \in \mathcal{A}$ with $F(\varphi)=\left\{\zeta_{1}, \ldots, \zeta_{r}\right\}$ and let $n_{j}=2 m_{j}$ denote the order of contact of $\varphi$ with the unit circle at $\zeta_{j}$. Then there exists a decomposition,

$$
C_{\varphi} \equiv C_{\varphi_{1}}+\ldots+C_{\varphi_{r}} \quad(\bmod \mathcal{K})
$$

where for each $j=1, \ldots, r, \varphi_{j}$ is a rational self-map of $\mathbb{D}$ of degree $m_{j}$ which maps $\zeta_{j}$ into the unit circle $\partial \mathbb{D}$ and the rest of $\partial \mathbb{D}$ into $\mathbb{D}$ and satisfies $D_{n_{j}}\left(\varphi_{j}, \zeta_{j}\right)=D_{n_{j}}\left(\varphi, \zeta_{j}\right)$.

Proof. Existence of $\varphi_{1}, \ldots, \varphi_{r}$ follows from Lemma 4.2.2. The result follows by applying Theorem 4.2 .1 to $\varphi, \varphi_{1}, \ldots \varphi_{n}$ with constants $1,-1, \ldots,-1$.

In the following chapters, we will require that the inducing rational maps have an additional property of regularity. We define basic functions as follows, and proceed to prove our second decomposition theorem where each term is a composition operator induced by a basic function.

Definition 4.1. A function $\varphi$ analytic on $\mathbb{D}$ is a basic function with contact at $\zeta$ if the following hold.

1. $\varphi$ is a rational function mapping the unit disk $\mathbb{D}$ into itself.

2. $\varphi(\zeta)$ is on the unit circle, and $\varphi$ maps the rest of the unit circle into $\mathbb{D}$.

3. $\varphi(\zeta)$ is a regular value for $\varphi$. 
Lemma 4.2.4. Let $\varphi \in \mathcal{A}$ and $\zeta \in F(\varphi)$. Let $n=2 m$ denote the order of contact of $\varphi$ with $\partial \mathbb{D}$ at $\zeta$. Then there exists a degree $m+1$ basic function $\varphi_{0}$ with order of contact $n$ with $\partial \mathbb{D}$ at $\zeta$ which satisfies $D_{n}\left(\varphi_{0}, \zeta\right)=D_{n}(\varphi, \zeta)$.

Proof. We begin as in the proof of Lemma 4.2.2, by defining $\lambda=\varphi(\zeta)$ and $f=$ $\tau_{\lambda} \circ \varphi \circ \tau_{\zeta}^{-1}$. Here, by Proposition 3.4.4, there exists a degree $m+1$ rational function $G \in \mathcal{P}_{0}$ that maps $\widehat{\mathbb{R}} \backslash\{0\}$ into $\mathbb{H}$, has initial Taylor coefficients $a_{0}, \ldots, a_{n}$ at $z=0$, and has $z=0$ as a regular value.

Again, we define $\varphi_{0}=\tau_{\lambda}^{-1} \circ G \circ \tau_{\zeta}$ and get that $\varphi_{0}$ is a degree $m+1$ rational function with the desired boundary behavior at $\zeta$. Since $D_{n}\left(\varphi_{0}, \zeta\right)$ is determined by $D_{n}\left(\tau_{\zeta}, \zeta\right)$, $D_{n}(G, 0)$ and $D_{n}\left(\tau_{\lambda}^{-1}, 0\right)$ (see Theorem 5.3.1), $\varphi_{0}$ satisfies $D_{n}\left(\varphi_{0}, \zeta\right)=D_{n}(\varphi, \zeta)$. Additionally, since $G$ has order of contact $n$ with $\mathbb{R}$ at 0 and by Proposition $2.4 .2, \varphi_{0}$ also has order of contact $n$ with $\partial \mathbb{D}$ at $\zeta$.

Now, since 0 is a regular value for $G$, we have that

$$
G(w)=\left(\tau_{\lambda} \circ \varphi_{0} \circ \tau_{\zeta}^{-1}\right)(w)=0
$$

has $m+1$ distinct solutions. So

$$
\varphi_{0}\left(\tau_{\zeta}^{-1}(w)\right)=\left(\tau_{\lambda}^{-1}\right)(0)=\lambda
$$

has $m+1$ distinct solutions, i.e., $\lambda$ is a regular value for $\varphi_{0}$.

Theorem 4.2.5. Let $\varphi \in \mathcal{A}$ with $F(\varphi)=\left\{\zeta_{1}, \ldots, \zeta_{r}\right\}$ and let $n_{j}=2 m_{j}$ denote the order of contact of $\varphi$ with the unit circle at $\zeta_{j}$. Then there exists a decomposition,

$$
C_{\varphi} \equiv C_{\varphi_{1}}+\ldots+C_{\varphi_{r}} \quad(\bmod \mathcal{K})
$$


where for each $j=1, \ldots, r, \varphi_{j}$ is a basic function of degree $m_{j}+1$ which has contact at $\zeta_{j}$ and satisfies $D_{n_{j}}\left(\varphi_{j}, \zeta_{j}\right)=D_{n_{j}}\left(\varphi, \zeta_{j}\right)$

Proof. Existence of $\varphi_{1}, \ldots, \varphi_{r}$ follows from Lemma 4.2.4. The result follows by applying Theorem 4.2 .1 to $\varphi, \varphi_{1}, \ldots \varphi_{n}$ with constants $1,-1, \ldots,-1$.

\subsection{Weighted Composition Operators and Adjoints in the Calkin Algebra}

In [19], it is shown that the coset of the weighted composition operator $M_{w} C_{\varphi}$ in the Calkin algebra is determined by the values of $w$ on $E(\varphi)$ in the following sense.

Proposition 4.3.1. [19, Theorem 3.1] Let $\varphi$ be an analytic self-map of $\mathbb{D}$ with $\left|\varphi\left(e^{i \theta}\right)\right|<1$ a.e. Suppose $w$ is a bounded measurable function on $\partial \mathbb{D}$ such that $|w|$ is continuous at every point of $E(\varphi)$. Then

$$
M_{w} C_{\varphi} \text { is compact if and only if } w \equiv 0 \text { on } E(\varphi) \text {. }
$$

Here we think of the operator $M_{w} C_{\varphi}$ as mapping $H^{2}$ to $L^{2}$, however it can also be considered as mapping $L^{2}$ to $L^{2}$ and if $w \in H^{\infty}$ as mapping $H^{2}$ to $H^{2}$.

For the special case that $\varphi \in \mathcal{A}$ has contact with $\partial \mathbb{D}$ at exactly one point, we have the following corollary.

Corollary 4.3.2. Let $\varphi \in \mathcal{A}$ be such that $F(\varphi)=\{\zeta\}$. Suppose $w$ is a bounded 
measurable function on $\partial \mathbb{D}$ such that $w$ is continuous at $\zeta$. Then

$$
M_{w} C_{\varphi} \equiv w(\zeta) C_{\varphi} \quad(\bmod \mathcal{K})
$$

Proof. Let $v(z):=w(z)-w(\zeta)$. Then $v$ is bounded on $\partial \mathbb{D}$, continuous at $\zeta$ and satisfies $v(\zeta)=0$ so by Proposition 4.3.1, $M_{v} C_{\varphi}=M_{w} C_{\varphi}-w(\zeta) C_{\varphi}$ is compact.

We will prove a similar result for weighted adjoints of composition operators. The proof relies on existence of an $H^{\infty}$ function which satisfies several boundary conditions. Before we turn to constructing this $H^{\infty}$ function, we state and prove the following fact.

Proposition 4.3.3. If $f \in L^{1}(\partial \mathbb{D})$ is real valued, then the map $h$ defined by

$$
h(z)=\int_{0}^{2 \pi} \frac{e^{i \theta}+z}{e^{i \theta}-z} f\left(e^{i \theta}\right) \frac{d \theta}{2 \pi},
$$

is analytic on $\mathbb{D}$, and has non-tangential boundary values a.e. on $\partial \mathbb{D}$ which satisfy

$$
\operatorname{Re} h\left(e^{i t}\right)=f\left(e^{i t}\right) \text { a.e. on } \partial \mathbb{D} \text {. }
$$

Furthermore, if $f$ is continuously differentiable on an open arc $\Gamma$ contained in $\partial \mathbb{D}$, then $h$ extends continuously to that arc.

Proof. We write

$$
h(z)=\int_{0}^{2 \pi} P_{z}\left(e^{i \theta}\right) f\left(e^{i \theta}\right) \frac{d \theta}{2 \pi}+i \int_{0}^{2 \pi} Q_{z}\left(e^{i \theta}\right) f\left(e^{i \theta}\right) \frac{d \theta}{2 \pi},
$$

where $P_{z}\left(e^{i \theta}\right)=\operatorname{Re} \frac{e^{i \theta}+z}{e^{i \theta}-z}$ and $Q_{z}\left(e^{i \theta}\right)=\operatorname{Im} \frac{e^{i \theta}+z}{e^{i \theta}-z}$ are the Poisson and the conjugate Poisson kernels. It is well known that the function

$$
u(z)=\int_{0}^{2 \pi} P_{z}\left(e^{i \theta}\right) f\left(e^{i \theta}\right) \frac{d \theta}{2 \pi}
$$


has non-tangential limits a.e. which satisfy

$$
\operatorname{Re} h\left(e^{i t}\right)=u\left(e^{i t}\right)=f\left(e^{i t}\right) \text { a.e. on } \partial \mathbb{D} \text {. }
$$

Furthermore, if $f$ is continuous on $\Gamma$ then, by standard techniques, the maps $u_{r}\left(e^{i t}\right)=$ $u\left(r e^{i t}\right)$ converge uniformly to their boundary function as $r \rightarrow 1$ on any closed arc contained in $\Gamma$. Since $u$ is the uniform limit of continuous functions on $\Gamma, u$ is also continuous on $\Gamma$. By [16, pp 78-79], similar results hold for the conjugate function $v$, defined by

$$
v(z)=\int_{0}^{2 \pi} Q_{z}\left(e^{i \theta}\right) f\left(e^{i \theta}\right) \frac{d \theta}{2 \pi} .
$$

Namely, $v$ has non-tangential limits a.e., and if $f$ is continuously differentiable on $\Gamma$, then the maps $v_{r}\left(e^{i t}\right)=v\left(r e^{i t}\right)$ converge uniformly to their boundary functions as $r \rightarrow 1$ on any closed arc contained in $\Gamma$. Since $v$ is the uniform limit of continuous functions on $\Gamma, v$ is also continuous on $\Gamma$.

Lemma 4.3.4. Let $\lambda \in \partial \mathbb{D}$ and $v$ be a non-negative bounded function on $\partial \mathbb{D}$ which is continuous at $\lambda$, continuously differentiable in a deleted neighborhood of $\lambda$ and satisfies $v(\lambda)=0$. Then there exists a function $b \in H^{\infty}$ which extends continuously to $\partial \mathbb{D}$ and satisfies $b(\lambda)=0$ and $\left|b\left(e^{i \theta}\right)\right| \geq v\left(e^{i \theta}\right)$ on $\partial \mathbb{D}$.

Proof. We begin by constructing a continuously differentiable real valued function $u$ on $\partial \mathbb{D}$. Let $\Gamma$ be a closed arc centered at $\lambda$ such that $v$ is continuously differentiable on $\Gamma \backslash\{\lambda\}$. We define $u$ on $\Gamma$ by

$$
u\left(e^{i \theta}\right)=v\left(e^{i \theta}\right)+\left|e^{i \theta}-\lambda\right|
$$


and extend $u$ to $\partial \mathbb{D} \backslash \Gamma$ so that the resulting function $u$ is continuously differentiable on $\partial \mathbb{D} \backslash\{\lambda\}$ and satisfies $u\left(e^{i \theta}\right) \geq v\left(e^{i \theta}\right)+\left|e^{i \theta}-\lambda\right|$ on $\partial \mathbb{D}$.

We let $f=\log u$, and note that $f$ is continuously differentiable on $\partial \mathbb{D} \backslash\{\lambda\}$ and satisfies $f\left(e^{i \theta}\right) \geq \log \left|e^{i \theta}-\lambda\right|$, so that $f \in L^{1}(\partial \mathbb{D})$. Therefore, by Proposition 4.3.3, $h$ defined by

$$
h(z)=\int_{0}^{2 \pi} \frac{e^{i \theta}+z}{e^{i \theta}-z} \log u\left(e^{i \theta}\right) \frac{d \theta}{2 \pi},
$$

is analytic on $\mathbb{D}$, and extends continuously to $\partial \mathbb{D} \backslash\{\lambda\}$. Furthermore, on $\partial \mathbb{D} \backslash\{\lambda\}$,

$$
\operatorname{Re} h\left(e^{i t}\right)=\log u\left(e^{i t}\right)
$$

We define $b$ on $\overline{\mathbb{D}}$ by $b(z)=e^{h(z)}$ for $z \neq \lambda$ and $b(\lambda)=0$. Then on $\partial \mathbb{D} \backslash\{\lambda\}$,

$$
\left|b\left(e^{i t}\right)\right|=e^{\operatorname{Re} h\left(e^{i t}\right)}=u\left(e^{i t}\right),
$$

so that $b \in H^{\infty}$ extends continuously to $\overline{\mathbb{D}} \backslash\{\lambda\}$, with $\left|b\left(e^{i t}\right)\right| \geq v\left(e^{i t}\right)$ on $\partial \mathbb{D}$. It remains to show that $b(z) \rightarrow 0$ as $z \rightarrow \lambda$ unrestrictedly in $\overline{\mathbb{D}}$.

Note that

$$
|b(z)|=\exp \left(\int_{0}^{2 \pi} P_{z}\left(e^{i \theta}\right) \log \left(u\left(e^{i \theta}\right)\right) \frac{d \theta}{2 \pi}\right)
$$

where $P_{z}\left(e^{i \theta}\right)=\operatorname{Re} \frac{e^{i \theta}+z}{e^{i \theta}-z}$ is the Poisson kernel. Thus, it suffices to show that

$$
\int_{0}^{2 \pi} P_{z}\left(e^{i \theta}\right) \log \left(u\left(e^{i \theta}\right)\right) \frac{d \theta}{2 \pi} \rightarrow-\infty
$$

as $z \rightarrow \lambda$ unrestrictedly in $\mathbb{D}$. Let $M>0$. We find $\epsilon$ such that for $|z-\lambda|<\epsilon$ the integral above is less than $-M$. Note that $\log u\left(e^{i \theta}\right) \rightarrow-\infty$ as $e^{i \theta} \rightarrow \lambda$, and let $\delta>0$ 
be such that on the $\operatorname{arc} \Gamma_{\delta}=\partial \mathbb{D} \cap\{|z-\lambda|<\delta\}$ we have $\log u\left(e^{i \theta}\right)<-4 M$. Then

$$
\int_{\Gamma_{\delta}} P_{z}\left(e^{i \theta}\right) \log \left(u\left(e^{i \theta}\right)\right) \frac{d \theta}{2 \pi}<-4 M \int_{\Gamma_{\delta}} P_{z}\left(e^{i \theta}\right) \frac{d \theta}{2 \pi}
$$

Note that $u$ is bounded from above so that $\log u<C$ for some constant $C$. Now choose $\epsilon<\delta$ such that for $|z-\lambda|<\epsilon$ we have both

$$
\int_{\Gamma_{\delta}} P_{z}\left(e^{i \theta}\right) \frac{d \theta}{2 \pi}>\frac{1}{2}
$$

and $P_{z}\left(e^{i \theta}\right)<\frac{M}{C}$ for all $e^{i \theta} \in \partial \mathbb{D} \backslash \Gamma_{\delta}$.

Then for all $z \in \mathbb{D}$ such that $|z-\lambda|<\epsilon$, we have

$$
\begin{aligned}
\int_{0}^{2 \pi} P_{z}\left(e^{i \theta}\right) \log u\left(e^{i \theta}\right) \frac{d \theta}{2 \pi} & =\int_{\Gamma_{\delta}} P_{z}\left(e^{i \theta}\right) \log u\left(e^{i \theta}\right) \frac{d \theta}{2 \pi}+\int_{\partial \mathbb{D} \backslash \Gamma_{\delta}} P_{z}\left(e^{i \theta}\right) \log u\left(e^{i \theta}\right) \frac{d \theta}{2 \pi} \\
& <-4 M \int_{\Gamma_{\delta}} P_{z}\left(e^{i \theta}\right) \frac{d \theta}{2 \pi}+\int_{\partial \mathbb{D} \backslash \Gamma_{\delta}} \frac{M}{C} \cdot C \frac{d \theta}{2 \pi} \\
& <-4 M \cdot \frac{1}{2}+M=-M .
\end{aligned}
$$

This completes the proof.

Proposition 4.3.5. Let $\varphi \in \mathcal{A}$ be such that $F(\varphi)=\{\zeta\}$. Suppose $w$ is a bounded measurable function on $\partial \mathbb{D}$ which is continuously differentiable in a deleted neighborhood of $\lambda=\varphi(\zeta)$. Then

$$
M_{w} C_{\varphi}^{*} \equiv w(\lambda) C_{\varphi}^{*} \quad(\bmod \mathcal{K})
$$

where $M_{w}$ is viewed as an operator from $H^{2}$ to $L^{2}$, and in particular,

$$
T_{w} C_{\varphi}^{*} \equiv w(\lambda) C_{\varphi}^{*} \quad(\bmod \mathcal{K})
$$


Proof. We can write

$$
M_{w} C_{\varphi}^{*}=\left(M_{w-w(\lambda)}+M_{w(\lambda)}\right) C_{\varphi}^{*}=M_{w-w(\lambda)} C_{\varphi}^{*}+w(\lambda) C_{\varphi}^{*} .
$$

Thus, it suffices to prove that $M_{v} C_{\varphi}^{*}$ is compact where $v=w-w(\lambda)$.

Note that $|v|$ is continuously differentiable in some deleted neighborhood of $\lambda$, and so by Lemma 4.3.4 there exists a $b \in H^{\infty}$ which extends continuously to $\partial \mathbb{D}$ and satisfies $b(\lambda)=0$ and $\left|b\left(e^{i \theta}\right)\right| \geq v\left(e^{i \theta}\right)$ on $\partial \mathbb{D}$. We get that for all $f \in H^{2}$,

$$
\begin{aligned}
\left\|M_{v} C_{\varphi}^{*} f\right\|^{2} & =\int_{0}^{2 \pi}\left|v\left(e^{i \theta}\right)\right|^{2}\left|\left(C_{\varphi}^{*} f\right)\left(e^{i \theta}\right)\right|^{2} \frac{d \theta}{2 \pi} \\
& \leq\left.\int_{0}^{2 \pi}\left|\overline{\left.b\left(e^{i \theta}\right)\right|^{2}}\right|\left(C_{\varphi}^{*} f\right)\left(e^{i \theta}\right)\right|^{2} \frac{d \theta}{2 \pi}=\left\|M_{\bar{b}} C_{\varphi}^{*} f\right\|^{2},
\end{aligned}
$$

so by Proposition 2.1.2, it suffices to show that $M_{\bar{b}} C_{\varphi}^{*}$ is a compact operator from $H^{2}$ to $L^{2}$. We write

$$
M_{\bar{b}} C_{\varphi}^{*}=P M_{\bar{b}} C_{\varphi}^{*}+(I-P) M_{\bar{b}} C_{\varphi}^{*}
$$

and show that both terms on the right hand side are compact.

First note that since $\bar{b}$ is a continuous function on $\partial \mathbb{D}$, the $L^{2}$ operator $(I-P) M_{\bar{b}} P$ is compact (see the version of Hartman's theorem in [22, p 214,Theorem 2.2.5]). Thus, the term $(I-P) M_{\bar{b}} C_{\varphi}^{*}=(I-P) M_{\bar{b}} P C_{\varphi}^{*}$ is compact from $H^{2}$ to $L^{2}$.

We now show $P M_{\bar{b}} C_{\varphi}^{*}=T_{\bar{b}} C_{\varphi}^{*}$ is compact on $H^{2}$ by looking at its adjoint $\left(T_{\bar{b}} C_{\varphi}^{*}\right)^{*}=$ $C_{\varphi} T_{b}$. Since $b \in H^{\infty}$, we have

$$
C_{\varphi} T_{b}=C_{\varphi} M_{b}=M_{b \circ \varphi} C_{\varphi}
$$


Since $\varphi \in \mathcal{A}$ with $F(\varphi)=\{\zeta\}$ and $b(\varphi(\zeta))=b(\lambda)=0$, by Corollary 4.3.2 we have

$$
M_{b \circ \varphi} C_{\varphi} \equiv b(\varphi(\zeta)) C_{\varphi} \equiv 0 \cdot C_{\varphi} \equiv 0 \quad(\bmod \mathcal{K})
$$

provided that $|b \circ \varphi|$ considered as a function on $\partial \mathbb{D}$ is continuous at $\zeta$. Recall that $b$ is continuous on $\overline{\mathbb{D}}$, and that $\varphi \in \mathcal{A}$ with $F(\varphi)=\{\zeta\}$ so that $\varphi\left(e^{i \theta}\right)$ is in $\mathbb{D}$ and tends to $\lambda$ as $e^{i \theta} \rightarrow \zeta$. Thus $|b \circ \varphi|$ is indeed continuous at $\zeta$. 


\section{Chapter 5}

\section{Adjoint Formula for Rationally Induced Composition Operators}

Recent work of Cowen-Gallardo [12], Hammond-Moorhouse-Robbins [15] and BourdonShapiro [7] has produced pointwise formulas for $C_{\varphi}^{*}$, where the inducing map $\varphi$ is rational. The constituent parts of these pointwise formulas contain multiple-valued analytic functions which do not necessarily represent well-defined operators individually. In Section 5.1 we show how to work with these pointwise formulas to produce legitimate operator equations involving $C_{\varphi}^{*}$ for the rational case. Then, in Section 5.2, we consider the case where $\varphi$ is basic and reduce our equations to the Calkin algebra.

\subsection{From Pointwise Formula To Operator Equa- tion}

Let $\varphi$ be a rational self-map of $\mathbb{D}$ of degree $d$. We associate with $\varphi$ its exterior map $\varphi_{e}:=\rho \circ \varphi \circ \rho$, where $\rho$ is the inversion in the unit circle defined by $\rho(z):=1 / \bar{z}$. Then $\varphi_{e}$ maps $\mathbb{D}_{e}:=\{z \in \mathbb{C}:|z|>1\}$ into itself, and so $\varphi_{e}^{-1}(\mathbb{D}) \subset \mathbb{D}$. 
Recall from Section 2.5 that for any simply connected domain $V$ consisting of regular values of $\varphi_{e}$, there exist $d$ distinct branches $\sigma_{1}, \ldots, \sigma_{d}$ of $\varphi_{e}^{-1}$ defined on $V$. Note that one possible choice of $V$ is the unit disk with radial slits from each critical value of $\varphi_{e}$ to the unit circle removed. A choice that may be much smaller but sufficient for our needs is a sufficiently small neighborhood of a regular value of $\varphi_{e}$.

The work of Cowen-Gallardo [12] and Hammond-Moorhouse-Robbins [15] has produced the following pointwise formula for the adjoint of a rationally-induced composition operator.

Proposition 5.1.1. [15, Corollary 8] Suppose that $V$ is a set on which d distinct branches $\sigma_{1}, \ldots, \sigma_{d}$ of $\varphi_{e}^{-1}$ are defined. Then for all $f \in H^{2}$ and all $z \in V \cap \mathbb{D}$,

$$
C_{\varphi}^{*} f(z)=\frac{f(0)}{1-\overline{\varphi(\infty)} z}+\sum_{j=1}^{d} \frac{z \sigma_{j}^{\prime}(z)}{\sigma_{j}(z)} f\left(\sigma_{j}(z)\right)
$$

In [7], Bourdon-Shapiro give an elementary proof for this original formula and introduce the following variant which we will use.

Proposition 5.1.2. [7, Corollary 8] Suppose that $V$ is a set on which d distinct branches $\sigma_{1}, \ldots, \sigma_{d}$ of $\varphi_{e}^{-1}$ are defined. Then for all $f \in H^{2}$ and all $z \in V \cap \mathbb{D}$,

$$
C_{\varphi}^{*} f(z)=\frac{f(0)}{1-\overline{\varphi(0)} z}+\sum_{j=1}^{d} z \sigma_{j}^{\prime}(z) S^{*} f\left(\sigma_{j}(z)\right)
$$

where $S^{*}$ is the adjoint of the shift operator $S$ defined by $(S f)(z)=z f(z)$. 


\subsubsection{Wishful Thinking - The Outer Regular Case}

Note that Eq. (5.1.1) can be rewritten, at least formally, as

$$
C_{\varphi}^{*}=\Lambda+\sum_{j=1}^{d} M_{h_{j}} C_{\sigma_{j}} S^{*},
$$

where $h_{j}(z)=z \sigma_{j}^{\prime}(z)$, and $\Lambda$ is the rank one operator defined by $\Lambda(f):=\frac{f(0)}{1-\overline{\varphi(0)} z}$. Notice that the terms $M_{h_{j}} C_{\sigma_{j}} S^{*}$ are reminiscent of Cowen's original formula for the linear-fractional case.

Bourdon-Shapiro define $\varphi$ as outer regular when its critical values all lie in $\mathbb{D}$. Note that $w \in \mathbb{C}$ is a regular value for $\varphi_{e}$ if and only if $\rho(w)$ is a regular value for $\varphi$, so that for outer regular functions $\varphi$ we can choose $V=r \mathbb{D}$ for some $r>1$. Then, restricting domains to $\mathbb{D}$, we have that $\sigma_{1}, \ldots, \sigma_{d}$ are analytic self-maps of $\mathbb{D}$ and $h_{1}, \ldots, h_{d}$ are $H^{\infty}$ functions. Thus, the wishful thinking equation Eq. (5.1.2) is a legitimate operator equation in the outer regular case [7, Theorem 13(a)].

Example 5.1. [7, Example 9] Let $\varphi$ be the analytic self-map of $\mathbb{D}$ fixing 1 defined by

$$
\varphi(z)=\frac{1}{3-z-z^{2}}
$$

Then $\varphi$ has a critical point at $z=-\frac{1}{2}$ so $\varphi\left(-\frac{1}{2}\right)=\frac{4}{13}$ is a critical value. Note that $\varphi(\infty)=0$ is also a critical since $\varphi^{-1}(\{0\})$ contains only one point. Since both critical values of $\varphi$ lie in $\mathbb{D}, \varphi$ is outer regular and so Eq. (5.1.2) is a legitimate operator equation.

We calculate $\varphi_{e}=\rho \circ \varphi \circ \rho$ and solve the equation $\varphi_{e}(w)=z$ to obtain $\sigma_{1}, \sigma_{2}$.

$$
\varphi_{e}(w)=3-\frac{1}{w}-\frac{1}{w^{2}}
$$




$$
\sigma_{1}(z)=\frac{1+\sqrt{13-4 z}}{2(3-z)} \quad \text { and } \quad \sigma_{1}(z)=\frac{1-\sqrt{13-4 z}}{2(3-z)}
$$

where $\sqrt{ }$ denotes the principal branch of the square root. Indeed, $\sigma_{1}$ and $\sigma_{2}$ are analytic on $\mathbb{C} \backslash\left[\frac{13}{4}, \infty\right)$ except for an isolated singularity at $z=3$.

Thus Eq. (5.1.2) provides the following legitimate operator formula for $C_{\varphi}^{*}$ :

$$
C_{\varphi}^{*}=\Lambda+M_{h_{1}} C_{\sigma_{1}} S^{*}+M_{h_{2}} C_{\sigma_{2}} S^{*}
$$

Note that $\widetilde{\varphi}=\tau_{1} \circ \varphi \circ \tau_{1}^{-1}$ is

$$
\widetilde{\varphi}(z)=\frac{i z^{2}-3 z}{2 z^{2}+5 i z-1}
$$

and has Taylor expansion

$$
\widetilde{\varphi}(z)=3 z+14 i z^{2}+O\left(z^{3}\right)
$$

and so $\varphi$ has order of contact 2 with the unit circle at $\zeta=1$.

The fact that the outer regular function in the example has order of contact 2 with the unit circle is no coincidence. We prove the following proposition using results from Section 5.3.

Proposition 5.1.3. If $\varphi$ is rational such that $\varphi$ has order of contact $n>2$ with the unit circle at $\zeta$, then $\varphi$ is not outer regular.

Proof. Suppose that $\varphi$ is outer regular and let $\sigma$ be a branch of $\varphi_{e}^{-1}$ mapping $\varphi(\zeta)$ to $\zeta$ and defined on $\mathbb{D}$. We transfer the problem to the upper half-plane and work with

$$
\widetilde{\varphi}=\tau_{\varphi(\zeta)}^{-1} \circ \varphi \circ \tau_{\zeta}, \quad \widetilde{\varphi_{e}}=\tau_{\varphi(\zeta)}^{-1} \circ \varphi_{e} \circ \tau_{\zeta}, \quad \widetilde{\sigma}=\tau_{\zeta}^{-1} \circ \sigma \circ \tau_{\varphi(\zeta)}
$$


which are all analytic self-maps of the upper half-plane according to our assumption.

Let $a_{0}, a_{1}, a_{2}, a_{3}$ denote the initial Taylor coefficients of $\widetilde{\varphi}$ at $z=0$, and $b_{0}, b_{1}, b_{2}, b_{3}$ denote those of $\widetilde{\sigma}$. In Proposition 5.3.6, we show that $\sigma$ has order of contact $n$ at $\lambda$. Thus by Theorem 3.1.3 the Hankel matrices $H_{m}\left(a_{1}, \ldots, a_{n-1}\right)$ and $H_{m}\left(b_{1}, \ldots, b_{n-1}\right)$ are both positive, and in particular, their 2 nd leading principal minors are positive, i.e.,

$$
a_{1} a_{3}-a_{2}^{2}>0, \quad b_{1} b_{3}-b_{2}^{2}>0
$$

In Proposition 5.3.4 we show that $\widetilde{\varphi}$ and $\widetilde{\varphi_{e}}$ have equal Taylor coefficient $a_{0}, \ldots, a_{n-1}$. Noting that $\widetilde{\varphi_{e}} \circ \sigma=i d$, we can therefore express $b_{1}, b_{2}, b_{3}$ in terms of $a_{1}, a_{2}, a_{3}$ using the product rule (or Faà di Bruno's formula, Theorem 5.3.1). We get the equations

$$
\begin{aligned}
& 1=\left(\widetilde{\varphi_{e}} \circ \widetilde{\sigma}\right)^{\prime}(z)=\widetilde{\varphi}_{e}^{\prime}(\widetilde{\sigma}(z)) \widetilde{\sigma}^{\prime}(z) \\
& 0=\left(\widetilde{\varphi_{e}} \circ \widetilde{\sigma}\right)^{\prime \prime}(z)=\widetilde{\varphi}_{e}^{\prime \prime}(\widetilde{\sigma}(z)) \widetilde{\sigma}^{\prime}(z)^{2}+\widetilde{\varphi}_{e}^{\prime}(\widetilde{\sigma}(z)) \widetilde{\sigma}^{\prime \prime}(z) \\
& 0=\left(\widetilde{\varphi_{e}} \circ \widetilde{\sigma}\right)^{\prime \prime \prime}(z)=\widetilde{\varphi}_{e}^{\prime \prime \prime}(\widetilde{\sigma}(z)) \widetilde{\sigma}^{\prime}(z)^{3}+3 \widetilde{\varphi}_{e}^{\prime \prime}(\widetilde{\sigma}(z)) \widetilde{\sigma}^{\prime}(z) \widetilde{\sigma}^{\prime \prime}(z)+\widetilde{\varphi}_{e}^{\prime}(\widetilde{\sigma}(z)) \widetilde{\sigma}^{\prime \prime \prime}(z),
\end{aligned}
$$

and evaluating at $z=0$, we have

$$
1=a_{1} b_{1}, \quad 0=2 a_{2} b_{1}^{2}+a_{1} \cdot 2 b_{2}, \quad 0=6 a_{3} b_{1}^{3}+3 \cdot 2 a_{2} b_{1} \cdot 2 b_{2}+a_{1} \cdot 6 b_{3} .
$$

Solving for $b_{1}, b_{2}, b_{3}$ we see that

$$
b_{1}=\frac{1}{a_{1}}, \quad b_{2}=-\frac{a_{2}}{a_{1}^{3}}, \quad b_{3}=\frac{2 a_{2}^{2}-a_{1} a_{3}}{a_{1}^{5}} .
$$

Thus

$$
b_{1} b_{3}-b_{2}^{2}=\frac{1}{a_{1}} \cdot \frac{2 a_{2}^{2}-a_{1} a_{3}}{a_{1}^{5}}-\frac{a_{2}^{2}}{a_{1}^{6}}=\frac{-\left(a_{1} a_{3}-a_{2}^{2}\right)}{a_{1}^{6}}<0
$$

which is a contradiction. 


\subsubsection{Operator Equation Involving $C_{\varphi}^{*}$}

In general, the set $V$ on which $\sigma_{1}, \ldots, \sigma_{d}$ are analytic can not be chosen to contain all of $\mathbb{D}$, and so the wishful thinking operators $C_{\sigma_{1}}, \ldots, C_{\sigma_{d}}$ in Eq. (5.1.2) are not legitimate operators. We overcome this difficulty by pre-composing with a map $\psi$ with image contained in $V \cap \mathbb{D}$ to obtain analytic self-maps of $\mathbb{D}, \sigma_{1} \circ \psi, \ldots, \sigma_{d} \circ \psi$. This will enable us to write a legitimate operator formula for $C_{\psi} C_{\varphi}^{*}$.

Proposition 5.1.4. Let $\psi$ be an analytic self-map of $\mathbb{D}$ satisfying $\overline{\psi(\mathbb{D})} \subset V$. Then

$$
C_{\psi} C_{\varphi}^{*}=C_{\psi} \Lambda+\sum_{j=1}^{d} M_{h_{j} \circ \psi} C_{\sigma_{j} \circ \psi} S^{*}
$$

where $h_{j}(z)=z \sigma_{j}^{\prime}(z), S^{*}$ is the adjoint of the shift operator and $\Lambda$ is the rank one operator defined by $\Lambda(f):=\frac{f(0)}{1-\overline{\varphi(0)} z}$.

In particular, $h_{j} \circ \psi$ are $H^{\infty}$ functions and $\sigma_{j} \circ \psi$ are analytic self-maps of $\mathbb{D}$.

Proof. Since $\psi$ maps $\mathbb{D}$ into $V$ and $\sigma_{1}, \ldots, \sigma_{d}$ are analytic on $V$, we get that $\sigma_{1} \circ$ $\psi, \ldots, \sigma_{d} \circ \psi$ are analytic self-maps of $\mathbb{D}$ (recall that $\left.\varphi_{e}^{-1}(\mathbb{D}) \subset \mathbb{D}\right)$. The functions $\sigma_{1}^{\prime}, \ldots, \sigma_{d}^{\prime}$ are analytic on $V$ and so bounded on $\psi(\mathbb{D})$, so $h_{1} \circ \psi, \ldots, h_{d} \circ \psi$ are $H^{\infty}$ functions. By the pointwise formula given in Proposition 5.1.2, for all $f \in H^{2}$ and all 
$z \in \mathbb{D}$ we have

$$
\begin{aligned}
C_{\psi} C_{\varphi}^{*} f(z)=C_{\varphi}^{*} f(\psi(z)) & =\frac{f(0)}{1-\overline{\varphi(0)} \psi(z)}+\sum_{j=1}^{d} \psi(z) \sigma_{j}^{\prime}(\psi(z)) S^{*} f\left(\sigma_{j}(\psi(z))\right) \\
& =(\Lambda f)(\psi(z))+\sum_{j=1}^{d} h_{j}(\psi(z)) S^{*} f\left(\left(\sigma_{j} \circ \psi\right)(z)\right) \\
& =\left(C_{\psi} \Lambda f\right)(z)+\sum_{j=1}^{d}\left(M_{h_{j} \circ \psi} C_{\sigma_{j} \circ \psi} S^{*} f\right)(z) .
\end{aligned}
$$

Therefore, the operator formula in the statement holds.

\subsection{The Basic Case}

We turn to the case where $\varphi$ is a basic function of degree $d$ with order of contact $n$ at $\zeta$. Denote $\lambda=\varphi(\zeta)$ and recall that $\lambda \in \partial \mathbb{D}$ is a regular value for $\varphi$ and so $\rho(\lambda)=\lambda$ is a regular value for $\varphi_{e}$. Thus we can choose $V$ to be a neighborhood of $\lambda$ consisting of regular values of $\varphi_{e}$.

Recall from Section 2.5 that $\sigma_{1}(V), \ldots, \sigma_{d}(V)$ are pairwise disjoint, and let $\sigma$ denote the unique branch of $\varphi_{e}^{-1}$ that maps $\lambda$ to $\zeta$.

Proposition 5.2.1. Let $\psi$ be an analytic self map of $\mathbb{D}$ satisfying $\overline{\psi(\mathbb{D})} \subset V$. Then

$$
C_{\psi} C_{\varphi}^{*} \equiv M_{h \circ \psi} \mathbb{C}_{\sigma \circ \psi} S^{*}(\bmod \mathcal{K})
$$

where $h(z):=z \sigma^{\prime}(z)$.

In particular, $h \circ \psi$ is an $H^{\infty}(\mathbb{D})$ function and $\sigma \circ \psi$ is an analytic self-map of $\mathbb{D}$. 
Proof. Notice that since $\rho$ is the identity on $\partial \mathbb{D}, \varphi_{e}$ maps exactly one point in $\partial \mathbb{D}$ (the point $\zeta$ ) into $\partial \mathbb{D}$ and so $\varphi_{e}^{-1}(\partial \mathbb{D})=\{\zeta\}$. We also have that the branches $\sigma_{1}, \ldots, \sigma_{d}$ map $\overline{\psi(\mathbb{D})}$ to pairwise disjoint subsets of $\overline{\mathbb{D}}$ (see Section 2.5), and these sets are closed as continuous images of compact sets. Since $\lambda \in \overline{\psi(\mathbb{D})}$ and by the definition of $\sigma$, we see that $\zeta \in \sigma(\overline{\psi(\mathbb{D})})$, and so for $\sigma_{j} \neq \sigma$, the closed set $\sigma(\overline{\psi(\mathbb{D})})$ does not intersect $\partial \mathbb{D}$. Thus, we have $\left\|\sigma_{j} \circ \psi\right\|_{\infty}<1$ and so the composition operator $\mathbb{C}_{\sigma_{j} \circ \psi}$ is compact.

Since $\Lambda$ in Proposition 5.1.4 is rank one, reducing Proposition 5.1.4 modulo the compacts gives

$$
C_{\psi} C_{\varphi}^{*} \equiv M_{h \circ \psi} C_{\sigma \circ \psi} S^{*} \quad(\bmod \mathcal{K})
$$

where $h$ is defined by $h(z)=z \sigma^{\prime}(z)$. Proposition 5.1.4 also shows that $h \circ \psi$ is an $H^{\infty}(\mathbb{D})$ function and $\sigma \circ \psi$ is an analytic self-map of $\mathbb{D}$.

By continuity of $\sigma$ and since $\sigma(\lambda)=\zeta$, there exists a neighborhood $V_{0} \subset V$ of $\lambda$ on which $\sigma$ is bounded away from zero.

Proposition 5.2.2. Let $\psi$ be an analytic self map of $\mathbb{D}$ mapping $\overline{\mathbb{D}}$ into $V_{0}$. Then

$$
C_{\psi} C_{\varphi}^{*} \equiv M_{g \circ \psi} \mathbb{C}_{\sigma \circ \psi} \quad(\bmod \mathcal{K})
$$

where $g(z):=\frac{z \sigma^{\prime}(z)}{\sigma(z)}$.

In particular, $g \circ \psi$ is an $H^{\infty}(\mathbb{D})$ function and $\sigma \circ \psi$ is an analytic self-map of $\mathbb{D}$.

Proof. By Proposition 5.2.1,

$$
C_{\psi} C_{\varphi}^{*} \equiv M_{h \circ \psi} C_{\sigma \circ \psi} S^{*} \bmod \mathcal{K}
$$


where $h$ is defined by $h(z)=z \sigma^{\prime}(z)$, and $h \circ \psi \in H^{\infty}(\mathbb{D})$ and $\sigma \circ \psi$ is an analytic self-map of $\mathbb{D}$. Note that for any $f \in H^{2}$ and any nonzero $z \in \mathbb{D}$, we have that

$$
\left(S^{*} f\right)(z)=\frac{f(z)-f(0)}{z}
$$

Thus, there exists a $K_{1} \in \mathcal{K}$ such that for any $f \in H^{2}$ and $z \in \mathbb{D}$, we have

$$
\begin{aligned}
\left(C_{\psi} C_{\varphi}^{*} f\right)(z) & =\left(M_{h \circ \psi} C_{\sigma \circ \psi} S^{*} f\right)(z)+\left(K_{1} f\right)(z) \\
& =\psi(z) \sigma^{\prime}(\psi(z)) \frac{f(\sigma(\psi(z)))-f(0)}{\sigma(\psi(z))}+\left(K_{1} f\right)(z) \\
& =\frac{\psi(z) \sigma^{\prime}(\psi(z))}{\sigma(\psi(z))} f(\sigma(\psi(z)))-\frac{\psi(z) \sigma^{\prime}(\psi(z))}{\sigma(\psi(z))} f(0)+\left(K_{1} f\right)(z) \\
& =g(\psi(z)) f(\sigma(\psi(z)))-g(\psi(z)) f(0)+\left(K_{1} f\right)(z)
\end{aligned}
$$

Since $\psi$ maps $\overline{\mathbb{D}}$ into $V_{0}, \sigma \circ \psi$ is bounded away from zero on $\overline{\mathbb{D}}$, and so $g \circ \psi$ is an $H^{\infty}(\mathbb{D})$ function. We can define the rank one operator $\left(K_{2} f\right)(z):=g(\psi(z)) f(0)$, and rewrite the above equation as

$$
\left(C_{\psi} C_{\varphi}^{*} f\right)(z)=\left(M_{g \circ \psi} C_{\sigma \circ \psi} f\right)(z)-\left(K_{2} f\right)(z)+\left(K_{1} f\right)(z)
$$

Thus $C_{\psi} C_{\varphi}^{*} f \equiv M_{g \circ \psi} C_{\sigma \circ \psi} \quad(\bmod \mathcal{K})$, as desired.

\subsubsection{Generalized Adjoint Formula in the Calkin Algebra}

If $\psi$ satisfies stronger conditions, we get the following formula which generalizes the adjoint formula modulo $\mathcal{K}$, developed by Kriete-MacCluer-Moorhouse [20], of a composition operator induced by a linear fractional map (see Proposition 2.3.8). 
Recall that $f(\xi)$ denotes the non-tangential limit of $f$ at $\xi$ for a function $f$ of $\mathbb{D}$ and $\xi \in \partial \mathbb{D}$.

Proposition 5.2.3. There exists a neighborhood $W$ of $\lambda$ such that for any self-map $\psi$ of $\mathbb{D}$ that satisfies:

1. $\psi(D) \subset W$

2. $\psi$ is analytic at $\lambda$ and fixes it

3. $\psi^{-1}(\{\lambda\}):=\{\beta \in \partial \mathbb{D}: \psi(\beta)$ exists and is equal to $\lambda\}=\{\lambda\}$

we have that the map $\sigma \circ \psi$ is in the class $\mathcal{A}$ with $F(\sigma \circ \psi)=\{\lambda\}$ and that

$$
C_{\psi} C_{\varphi}^{*} \equiv \frac{1}{\left|\varphi^{\prime}(\zeta)\right|} C_{\sigma \circ \psi} \quad(\bmod \mathcal{K})
$$

Proof. Let $W$ be a neighborhood of $\lambda$ such that the closure of $W$ is contained in $V_{0}$. Then for any $\psi$ as above, $\overline{\psi(\mathbb{D})}$ is contained in $V_{0}$, and so by Proposition 5.2 .2 we have

$$
C_{\psi} C_{\varphi}^{*} \equiv M_{g \circ \psi} C_{\sigma \circ \psi} \quad(\bmod \mathcal{K})
$$

To show that $\sigma \circ \psi \in \mathcal{A}$, first recall that

$$
E(\sigma \circ \psi)=\overline{\bigcup_{|\alpha|=1} s p t\left(\mu_{\alpha}^{s}\right)}
$$

and that for each $\alpha \in \partial \mathbb{D}, \mu_{\alpha}^{s}$ is carried by

$$
(\sigma \circ \psi)^{-1}(\{\alpha\})=\{\beta \in \partial \mathbb{D}:(\sigma \circ \psi)(\beta) \text { exists and is equal to } \alpha\} \text {. }
$$


Suppose $(\sigma \circ \psi)(\beta)=\alpha$ for some $\alpha, \beta \in \partial \mathbb{D}$. Then applying $\varphi_{e}$ we get that $\psi(\beta)$ exists and $\sigma(\psi(\beta))=\alpha$. Recall that $\varphi_{e}^{-1}(\partial \mathbb{D})=\{\zeta\}, \sigma(\lambda)=\zeta$ and $\sigma$ is univalent on $V$. Thus $\sigma(\psi(\beta))=\alpha$ implies that $\alpha=\zeta$ and $\psi(\beta)=\lambda$, and so by the third condition on $\psi$ we have $\beta=\lambda$. Therefore $\operatorname{spt}\left(\mu_{\alpha}^{s}\right)$ is empty for $\alpha \neq \zeta$, and $\operatorname{spt}\left(\mu_{\zeta}^{s}\right) \subset\{\lambda\}$. We conclude that $E(\sigma \circ \psi) \subset\{\lambda\}$.

We have that $\sigma \circ \psi$ is analytic at $\lambda$ and maps $\lambda$ to $\zeta$, so that $F(\sigma \circ \psi)=\{\lambda\}$. Since $\sigma \circ \psi$ is analytic as $\lambda$ and does not map an arc of $\partial \mathbb{D}$ containing $\lambda$ into $\partial \mathbb{D}$, $\sigma \circ \psi$ has finite order of contact at $\lambda$ and a Taylor expansion to that order about $\lambda$. This shows that $\sigma \circ \psi$ is indeed in the class $\mathcal{A}$.

Recall that due to the definition of $V_{0}$, for $g(z)=\frac{z \sigma^{\prime}(z)}{\sigma(z)}$ the map $g \circ \psi$ is an $H^{\infty}(\mathbb{D})$ function. Since $g \circ \psi$ is also continuous at $\lambda$, we can apply Corollary 4.3 .2 to get:

$$
C_{\psi} C_{\varphi}^{*} \equiv(g \circ \psi)(\lambda) C_{\sigma \circ \psi} \quad(\bmod \mathcal{K})
$$

We calculate

$$
(g \circ \psi)(\lambda)=g(\lambda)=\frac{\lambda \sigma^{\prime}(\lambda)}{\sigma(\lambda)}=\frac{\lambda}{\zeta \varphi^{\prime}(\zeta)}=\frac{1}{\zeta \bar{\lambda} \varphi^{\prime}(\zeta)},
$$

where $\sigma^{\prime}(\lambda)=1 / \varphi^{\prime}(\zeta)$ follows by applying the chain rule for $\sigma \circ \varphi_{e}$ at $z=\zeta$, and substituting $\varphi_{e}^{\prime}(\zeta)=\varphi^{\prime}(\zeta)$ which holds by Proposition 5.3.4 or direct calculation.

To complete the proof, note that by Theorem $2.3 .3, \varphi^{\prime}(\zeta)=d(\zeta) \bar{\zeta} \lambda$, where $d(\zeta)>$ 0 , and so $\left|\varphi^{\prime}(\zeta)\right|=d(\zeta)=\zeta \bar{\lambda} \varphi^{\prime}(\zeta)$.

Note that for a linear fractional map $\varphi(z)=\frac{a z+b}{c z+d}$, we have that $\varphi_{e}(z)$ is invertible, and $\varphi_{e}^{-1}(z)=\sigma(z)=\frac{\bar{a} z-\bar{c}}{-\bar{b} z+\bar{d}}$ is the Krein adjoint of $\varphi$. Recall that by 
Proposition 2.3.8,

$$
C_{\varphi}^{*} \equiv \frac{1}{\left|\varphi^{\prime}(\zeta)\right|} C_{\sigma} \quad(\bmod \mathcal{K})
$$

and so Proposition 5.2.3 can be viewed as a generalization of Proposition 2.3.8.

Note also that if $\tau \in \mathcal{A}$ with $F(\tau)=\{\zeta\}$, Proposition 2.3.8 can be extended to produce a formula for $C_{\tau}^{*}$ provided $\tau$ has order of contact 2 with $\partial \mathbb{D}$ at $\zeta$, whereas Proposition 5.2.3 can be extended to produce a formula for $C_{\psi} C_{\tau}^{*}$ provided that $\psi$ satisfies the conditions of Proposition 5.2.3, and regardless of the order of contact of $\tau$ with $\partial \mathbb{D}$ at $\zeta$.

\subsection{Relationship Between $\varphi$ and $\sigma$}

Let $\varphi$ be a rational function with order of contact $n$ with the unit circle at $\zeta$, mapping $\zeta$ to $\lambda$. Let $\sigma$ be a branch of $\varphi_{e}^{-1}$ mapping $\lambda$ to $\zeta$. Then $\sigma \circ \varphi_{e}=i d$ near $\zeta$ and $\varphi_{e} \circ \sigma=i d$ near $\lambda$. Thus

$$
D_{n}\left(\sigma \circ \varphi_{e}, \zeta\right)=(\zeta, 1,0, \ldots, 0), \quad \text { and } \quad D_{n}\left(\varphi_{e} \circ \sigma, \lambda\right)=(\lambda, 1,0, \ldots, 0)
$$

Although $\varphi$ and $\sigma$ are not inverse functions, we will show that they are "almost inverse" in the sense that

$$
D_{n-1}(\sigma \circ \varphi, \zeta)=(\zeta, 1,0, \ldots, 0), \quad \text { and } \quad D_{n-1}(\varphi \circ \sigma, \lambda)=(\lambda, 1,0, \ldots, 0)
$$

Throughout this section, we will use Faà di Bruno's formula, an identity generalizing the chain rule that has been known since 1800. The following is the statement of the formula in combinatorial form. 
Theorem 5.3.1 (Faà di Bruno's formula). [17] If $g$ is analytic at $z$ and $f$ is analytic at $g(z)$, then

$$
(f \circ g)^{(k)}(z)=\sum_{\pi \in \Pi} f^{(|\pi|)}(g(z)) \cdot \prod_{B \in \pi} g^{(|B|)}(z),
$$

where $\Pi$ is the set of partitions of $\{1, \ldots, k\}$.

Note that as a consequence of Theorem 5.3.1, if $g$ is analytic at $z$ and $f$ is analytic at $g(z)$ then $D_{k}(g, z)$ and $D_{k}(f, g(z))$ determine $D_{k}(f \circ g, z)$. We rewrite Faà di Bruno's formula in the following way.

Lemma 5.3.2. If $g$ is analytic at $z$ and $f$ is analytic at $g(z)$, then

$$
(f \circ g)^{(k)}(z)=f^{(k)}(g(z)) g^{\prime}(z)^{k}+F\left(D_{k-1}(f, g(z)), D_{k-1}(g, z)\right)+f^{\prime}(g(z)) g^{(k)}(z),
$$

where

$$
F\left(\left(a_{0}, a_{1}, \ldots, a_{k-1}\right),\left(b_{0}, b_{1}, \ldots, b_{k-1}\right)\right)=\sum_{\substack{\pi \in \Pi \\ 1<|\pi|<k}} a_{|\pi|} \cdot \prod_{B \in \pi} b_{|B|} .
$$

Proof. This follows from Theorem 5.3.1, with the first term originating from the partition $\pi=\{\{1\},\{2\}, \ldots,\{k\}\}$, and the last term originating from the partition $\pi=\{\{1,2, \ldots, k\}\}$

We transfer $\varphi, \varphi_{e}$ and $\sigma$ to the upper half-plane $\mathbb{H}=\{z \in \mathbb{C}: \operatorname{Im} z>0\}$, and analyze their relationships there. The corresponding self-maps of $\mathbb{H}$ are

$$
\widetilde{\varphi}=\tau_{\lambda} \circ \varphi \circ \tau_{\zeta}^{-1}, \quad \widetilde{\varphi_{e}}=\tau_{\lambda} \circ \varphi_{e} \circ \tau_{\zeta}^{-1}, \quad \widetilde{\sigma}=\tau_{\zeta} \circ \sigma \circ \tau_{\lambda}^{-1}
$$

where $\tau_{\alpha}(z)=i \frac{\alpha-z}{\alpha+z}$ is a conformal map which maps $\mathbb{D}$ onto $\mathbb{H}$ and $\alpha$ to 0 . Note that $\widetilde{\varphi}, \widetilde{\varphi_{e}}$ and $\widetilde{\sigma}$ all fix 0 and are analytic in a neighborhood of 0 
Lemma 5.3.3. $\widetilde{\varphi_{e}}$ is the upper half-plane exterior map associated with $\widetilde{\varphi}$, i.e.,

$$
\widetilde{\varphi_{e}}(z)=\overline{\widetilde{\varphi}(\bar{z})}
$$

Proof. Recall that $\varphi_{e}=\rho \circ \varphi \circ \rho$ where $\rho: z \rightarrow 1 / \bar{z}$, and so

$$
\widetilde{\varphi_{e}}(z)=\left(\tau_{\lambda} \circ \rho \circ \varphi \circ \rho \circ \tau_{\zeta}^{-1}\right)(z)
$$

We denote $r(z)=\bar{z}$, so that

$$
\overline{\widetilde{\varphi}(\bar{z})}=(r \circ \widetilde{\varphi} \circ r)(z)=\left(r \circ \tau_{\lambda} \circ \varphi \circ \tau_{\zeta}^{-1} \circ r\right)(z)
$$

It therefore suffices to show that $\tau_{\alpha} \circ \rho=r \circ \tau_{\alpha}$ for any $\alpha \in \partial \mathbb{D}$. Indeed

$$
\tau_{\alpha}\left(\frac{1}{\bar{z}}\right)=i \frac{\alpha-\frac{1}{\bar{z}}}{\alpha+\frac{1}{\bar{z}}}=i \frac{\bar{z}-\bar{\alpha}}{\bar{z}+\bar{\alpha}}=-i \frac{\bar{\alpha}-\bar{z}}{\bar{\alpha}+\bar{z}}=\overline{\tau_{\alpha}(z)}
$$

Proposition 5.3.4. $D_{n-1}(\varphi, \zeta)=D_{n-1}\left(\varphi_{e}, \zeta\right)$ and $\varphi^{(n)}(\zeta) \neq \varphi_{e}^{(n)}(\zeta)$.

Proof. By Theorem 5.3.1, it suffices show that

$$
D_{n-1}(\widetilde{\varphi}, 0)=D_{n-1}\left(\widetilde{\varphi_{e}}, 0\right) \text { and } \quad \widetilde{\varphi}^{(n)}(0) \neq \widetilde{\varphi}_{e}^{(n)}(0)
$$

Let $\widetilde{\varphi}(z)=\sum_{0}^{\infty} a_{k} z^{k}$ be the Taylor expansion of $\widetilde{\varphi}$ about 0 . Then by Lemma 5.3.3, we can write $\widetilde{\varphi_{e}}(z)=\overline{\widetilde{\varphi}(\bar{z})}=\sum_{0}^{\infty} \overline{a_{k}} z^{k}$, and so we have

$$
\widetilde{\varphi}^{(k)}(0)=k ! a_{k} \quad \text { and } \quad \widetilde{\varphi}_{e}^{(k)}(0)=k ! \overline{a_{k}}
$$

for all $k$. To complete the proof, recall that $\varphi$ has order of contact $n$ with the unit circle, and so $a_{0}, a_{1}, \ldots, a_{n-1} \in \mathbb{R}$ and $\operatorname{Im} a_{n}>0$. 
Lemma 5.3.5. The $n$ 'th order data for $\sigma \circ \varphi$ and $\varphi \circ \sigma$ is given by

$$
\begin{aligned}
& D_{n}(\sigma \circ \varphi, \zeta)=\left(\zeta, 1,0, \ldots, 0, c \cdot \sigma^{\prime}(\lambda)\right) \\
& D_{n}(\varphi \circ \sigma, \lambda)=\left(\lambda, 1,0, \ldots, 0, c \cdot \sigma^{\prime}(\lambda)^{n}\right),
\end{aligned}
$$

where $c$ is a non zero constant given by $c=\varphi^{(n)}(\zeta)-\varphi_{e}^{(n)}(\zeta)$.

Proof. By Lemma 5.3.2, $(\sigma \circ \varphi)^{(n)}(\zeta)$ and $\left(\sigma \circ \varphi_{e}\right)^{(n)}(\zeta)$ can be written as follows.

$$
\begin{gathered}
(\sigma \circ \varphi)^{(n)}(\zeta)=\sigma^{(n)}(\lambda) \varphi^{\prime}(\zeta)^{n}+F\left(D_{n-1}(\sigma, \lambda), D_{n-1}(\varphi, \zeta)\right)+\sigma^{\prime}(\lambda) \varphi^{(n)}(\zeta) \\
\left(\sigma \circ \varphi_{e}\right)^{(n)}(\zeta)=\sigma^{(n)}(\lambda) \varphi_{e}^{\prime}(\zeta)^{n}+F\left(D_{n-1}(\sigma, \lambda), D_{n-1}\left(\varphi_{e}, \zeta\right)\right)+\sigma^{\prime}(\lambda) \varphi_{e}^{(n)}(\zeta)
\end{gathered}
$$

Recall that $\sigma \circ \varphi_{e}=i d$ and so $\left(\sigma \circ \varphi_{e}\right)^{(n)}(\zeta)=0$, and that by Proposition 5.3.4 $D_{n-1}(\varphi, \zeta)=D_{n-1}\left(\varphi_{e}, \zeta\right)$. Thus subtracting the above equations yields:

$$
(\sigma \circ \varphi)^{(n)}(\zeta)=\sigma^{\prime}(\lambda) \cdot\left(\varphi^{(n)}(\zeta)-\varphi_{e}^{(n)}(\zeta)\right)
$$

Similarly, applying Theorem 5.3.1 to $(\varphi \circ \sigma)^{(n)}(\lambda)$ and $\left(\varphi_{e} \circ \sigma\right)^{(n)}(\lambda)$, we get

$$
\begin{gathered}
(\varphi \circ \sigma)^{(n)}(\zeta)=\varphi^{(n)}(\zeta) \sigma^{\prime}(\lambda)^{n}+F\left(D_{n-1}(\varphi, \zeta), D_{n-1}(\sigma, \lambda)\right)+\varphi^{\prime}(\zeta) \sigma^{(n)}(\lambda) \\
\left(\varphi_{e} \circ \sigma\right)^{(n)}(\zeta)=\varphi_{e}^{(n)}(\zeta) \sigma^{\prime}(\lambda)^{n}+F\left(D_{n-1}\left(\varphi_{e}, \zeta\right), D_{n-1}(\sigma, \lambda)\right)+\varphi_{e}^{\prime}(\zeta) \sigma^{(n)}(\lambda)
\end{gathered}
$$

Again, since $D_{n-1}(\varphi, \zeta)=D_{n-1}\left(\varphi_{e}, \zeta\right)$ and $\left(\sigma \circ \varphi_{e}\right)^{(n)}(\zeta)=0$, subtracting the above equations yields:

$$
(\varphi \circ \sigma)^{(n)}(\zeta)=\sigma^{\prime}(\lambda)^{n} \cdot\left(\varphi^{(n)}(\zeta)-\varphi_{e}^{(n)}(\zeta)\right)
$$

Recall that by Proposition 5.3.4, $\varphi_{e}^{(n)}(\zeta) \neq \varphi^{(n)}(\zeta)$, and so $c \neq 0$. 
As additional applications of Faà di Bruno's Formula, we calculate the order of contact of $\sigma$ with $\partial \mathbb{D}$ at $\lambda$ and determine the order of contact of composite maps under some assumptions.

Proposition 5.3.6. The map $\sigma$ has order of contact $n$ with $\partial \mathbb{D}$ at $\lambda$.

Proof. Let $\widetilde{\varphi}(z)=\sum_{0}^{\infty} a_{k} z^{k}$ and $\widetilde{\sigma}(z)=\sum_{0}^{\infty} b_{k} z^{k}$ be the Taylor expansions about 0 of $\widetilde{\varphi}$ and $\tilde{\sigma}$ respectively. We have that $a_{0}, \ldots, a_{n-1} \in \mathbb{R}$ and $\operatorname{Im} a_{n}>0$ since $\varphi$ has order of contact $n$ with $\mathbb{D}$, and it suffices to show that $b_{0}=0, b_{1}, \ldots, b_{n-1} \in \mathbb{R}$ and $\operatorname{Im} b_{n}>0$ (see Proposition 2.4.2, Proposition 2.4.1)

Recall that $\widetilde{\varphi_{e}}(z)=\overline{\widetilde{\varphi}(\bar{z})}=\sum_{0}^{\infty} \overline{a_{k}} z^{k}$ by Lemma 5.3.3. Notice that $\widetilde{\varphi}$ fixes 0 and maps $W \cap \mathbb{H}$ into $\mathbb{H}$ for some neighborhood $W$ of 0 , and so $a_{1}>0$ and $b_{1}=\widetilde{\sigma}^{\prime}(0)=$ $1 / \widetilde{\varphi_{e}}(0)=1 / a_{1}>0$. Using Lemma 5.3.2 for $\widetilde{\sigma} \circ \widetilde{\varphi_{e}}=i d$ and $k>1$, we get

$$
0=\widetilde{\sigma}^{(k)}(0) \widetilde{\varphi}_{e}^{\prime}(0)^{k}+F\left(D_{k-1}(\widetilde{\sigma}, 0), D_{k-1}\left(\widetilde{\varphi_{e}}, 0\right)\right)+\widetilde{\sigma}^{\prime}(0) \widetilde{\varphi}_{e}^{(k)}(0)
$$

Thus, by induction on $k=2, \ldots, n-1$, we see that $b_{0}, \ldots, b_{k}$ are real valued. For $k=n$, taking imaginary parts we get

$$
0=\operatorname{Im}\left(\widetilde{\sigma}^{(n)}(0)\right) \widetilde{\varphi}_{e}^{\prime}(0)^{n}+\widetilde{\sigma}^{\prime}(0) \operatorname{Im}\left(\widetilde{\varphi}_{e}^{(n)}(0)\right)
$$

or

$$
\operatorname{Im}\left(b_{n}\right)=-\frac{b_{1}}{a_{1}^{n}} \operatorname{Im}\left(\overline{a_{n}}\right)=\frac{b_{1}}{a_{1}^{n}} \operatorname{Im}\left(a_{n}\right)>0 .
$$


Proposition 5.3.7. Let $\psi_{1}$ be analytic at $\alpha_{1}$ such that for some neighborhood $W_{1}$ of $\alpha_{1}, \psi_{1}$ maps $W_{1} \cap \mathbb{D}$ into $\mathbb{D}$, and let $\psi_{2}$ be analytic at $\alpha_{2}=\psi_{1}\left(\alpha_{1}\right)$ such that for some neighborhood $W_{2}$ of $\alpha_{2}, \psi_{2}$ maps $W_{2} \cap \mathbb{D}$ into $\mathbb{D}$.

We define $N_{1}$ and $N_{2}$ by

$$
N_{i}= \begin{cases}c & \psi_{i} \text { has order of contact } c \text { with } \partial \mathbb{D} \text { at } \alpha_{i} \\ \infty & \psi_{i} \text { maps an arc of } \partial \mathbb{D} \text { containing } \alpha_{i} \text { into } \partial \mathbb{D}\end{cases}
$$

Let $K$ be the minimum of $M$ and $N$. Then if $K$ is finite, $\psi_{2} \circ \psi_{1}$ has order of contact $K$ at $\alpha_{1}$, and if $K$ is infinite then $\psi_{2} \circ \psi_{1}$ maps an arc of $\partial \mathbb{D}$ containing $\alpha_{1}$ into $\partial \mathbb{D}$.

Proof. The corresponding maps on the upper half-plane are

$$
f=\widetilde{\psi_{2}}=\tau_{\psi_{2}\left(\alpha_{2}\right)} \circ \psi_{2} \circ \tau_{\alpha_{2}}^{-1}, \quad g=\widetilde{\psi_{1}}=\tau_{\alpha_{2}} \circ \psi_{1} \circ \tau_{\alpha_{1}}^{-1}
$$

and

$$
\left(\widetilde{\psi_{2} \circ \psi_{1}}\right)=\tau_{\left(\psi_{2} \circ \psi_{1}\right)\left(\alpha_{1}\right)} \circ \psi_{2} \circ \psi_{1} \circ \tau_{\alpha_{1}}^{-1}=f \circ g .
$$

Note that these maps are all analytic in a neighborhood of 0 , and let $f(z)=\sum_{j=0}^{j=\infty} c_{j} z^{j}$ and $g(z)=\sum_{j=0}^{j=\infty} d_{j} z^{j}$ be the Taylor series of $f$ and $g$ there. Note that if $N_{2}$ is finite then by Proposition 2.4.2 and Proposition 2.4.1, $c_{1}, \ldots, c_{N-1} \in \mathbb{R}$ and $c_{N} \in \mathbb{H}$ and if $N_{2}$ is infinite then $c_{1}, c_{2}, \ldots \in \mathbb{R}$. The corresponding statement for $g$ holds similarly. Note that since $f$ and $g$ both map some neighborhood of zero intersected with $\mathbb{H}$ into $\mathbb{H}$, we have that $c_{1}, d_{1}>0$.

If $c_{1}, \ldots, c_{k}, d_{1}, \ldots, d_{k} \in \mathbb{R}$, then by Theorem 5.3 .1 and induction on $j=1, \ldots, k$, we see that $(f \circ g)^{\prime}(0), \ldots,(f \circ g)^{(j)}(0)$ are all real valued. Thus, $(f \circ g)^{\prime}(0), \ldots,(f \circ g)^{(k)}(0)$ 
are real valued in this case.

In the case where $K$ is infinite, we get that all Taylor coefficients of $f \circ g$ at 0 are real valued and so $f \circ g$ maps an interval in $\mathbb{R}$ containing 0 into $\mathbb{R}$. Thus $\psi_{2} \circ \psi_{1}$ maps an arc of $\partial \mathbb{D}$ containing $\alpha_{1}$ into $\partial \mathbb{D}$.

In the case where $K$ is finite, we get by the argument above that

$$
c_{1}, \ldots, c_{K-1}, d_{1}, \ldots, d_{K-1} \in \mathbb{R}
$$

By Lemma 5.3.2 at $z=0$ we have

$$
(f \circ g)^{(K)}(0)=f^{(K)}(0) g^{\prime}(0)^{+} F\left(D_{K-1}(f, 0), D_{K-1}(g, 0)\right)+f^{\prime}(0) g^{(K)}(0) .
$$

and taking imaginary parts we get

$$
\operatorname{Im}(f \circ g)^{(K)}(0)=g^{\prime}(0)^{k} \operatorname{Im} f^{(k)}(0)+f^{\prime}(0) \operatorname{Im} g^{(k)}(0)
$$

Since $c_{1}, d_{1}>0$, and $\operatorname{Im} c_{K}, \operatorname{Im} d_{K} \geq 0$ with at least one of them positive by the definition of $K$, it follows that $\operatorname{Im}(f \circ g)^{(K)}(0)>0$. Thus $f \circ g$ has order of contact $K$ with $\mathbb{R}$ at 0 , and so by Proposition 2.4.2, $\psi_{2} \circ \psi_{1}$ has order of contact $K$ at $\alpha_{1}$. 


\section{Chapter 6}

\section{Essential normality}

We begin by applying our results from Section 5.2 to characterize essential normality for the basic case. We then decompose $C_{\varphi}$ into a sum of basic composition operators and show that essential normality can only occur when this sum consists of a single term. Lastly, using the parametrization of functions with given order of contact proved in Chapter 3, we construct essentially normal composition operators which have arbitrary even order of contact with the unit circle at one point.

Definition 6.1. For $\epsilon>0$ we define $\psi_{\lambda, \epsilon}$ to be the Riemann mapping from $\mathbb{D}$ onto $\{|z-\lambda|<\epsilon\} \cap \mathbb{D}$, which fixes $\lambda$. Note that $\psi=\psi_{\lambda, \epsilon}$ extends continuously to $\partial \mathbb{D}$ and analytically across $\partial \mathbb{D}$ in a neighborhood of $\lambda$.

\subsection{Essential Normality For Basic Composition Op- erators}

Let $\varphi$ be a basic function with contact at $\zeta$ which fixes $\zeta$. Let $n$ be the order of contact

of $\varphi$ with $\partial \mathbb{D}$ at $\zeta$, and let $\sigma$ be the unique branch of $\varphi_{e}^{-1}$ defined on a neighborhood 
of $\zeta$ which fixes $\zeta$.

Proposition 6.1.1. There exists $\epsilon>0$ such that for $\psi=\psi_{\zeta, \epsilon}$, the following conditions are equivalent.

1. $C_{\psi}\left[C_{\varphi}, C_{\varphi}^{*}\right]$ is compact

2. $D_{n}(\sigma \circ \varphi \circ \psi, \zeta)=D_{n}(\varphi \circ \sigma \circ \psi, \zeta)$

3. $D_{n}(\sigma \circ \varphi, \zeta)=D_{n}(\varphi \circ \sigma, \zeta)$

4. $\varphi^{\prime}(\zeta)=1$

Proof. (1) $\Longleftrightarrow$ (2): Let $W$ be the neighborhood of $\zeta$ guaranteed by Proposition 5.2.3. Notice that $\varphi^{-1}(W) \cap W$ is open and contains $\zeta$, and choose $\epsilon>0$ such that $\{|z-\zeta| \leq \epsilon\} \cap \mathbb{D}$ is contained in $\varphi^{-1}(W) \cap W$.

Denote $\psi=\psi_{\zeta, \epsilon}$ and note that both $\psi$ and $\varphi \circ \psi$ map $\mathbb{D}$ into $W$, are analytic at $\zeta$ and fix $\zeta$. Additionally, both $\psi$ and $\varphi$ extend continuously to $\overline{\mathbb{D}}$ and satisfy

$$
\varphi^{-1}(\{\zeta\})=\{\zeta\}, \quad(\varphi \circ \psi)^{-1}(\{\zeta\})=\{\zeta\}
$$

Thus, by Proposition 5.2.3, both of the following equations in the Calkin algebra hold:

$$
C_{\psi} C_{\varphi}^{*} \equiv \frac{1}{\left|\varphi^{\prime}(\zeta)\right|} C_{\sigma \circ \psi} \quad(\bmod \mathcal{K}), \quad C_{\varphi \circ \psi} C_{\varphi}^{*} \equiv \frac{1}{\left|\varphi^{\prime}(\zeta)\right|} C_{\sigma \circ \varphi \circ \psi} \quad(\bmod \mathcal{K}),
$$


Therefore, we can express $C_{\psi}\left[C_{\varphi}, C_{\varphi}^{*}\right]$ in the Calkin algebra by

$$
\begin{aligned}
C_{\psi}\left[C_{\varphi}, C_{\varphi}^{*}\right] & \equiv C_{\psi} C_{\varphi} C_{\varphi}^{*}-C_{\psi} C_{\varphi}^{*} C_{\varphi} \equiv C_{\varphi \circ \psi} C_{\varphi}^{*}-C_{\psi} C_{\varphi}^{*} C_{\varphi} \\
& \equiv \frac{1}{\left|\varphi^{\prime}(\zeta)\right|} C_{\sigma \circ \varphi \circ \psi}-\frac{1}{\left|\varphi^{\prime}(\zeta)\right|} C_{\sigma \circ \psi} C_{\varphi} \equiv \frac{1}{\left|\varphi^{\prime}(\zeta)\right|}\left(C_{\sigma \circ \varphi \circ \psi}-C_{\varphi \circ \sigma \circ \psi}\right) \quad(\bmod \mathcal{K}) .
\end{aligned}
$$

Note that $\psi$ maps an arc of $\partial \mathbb{D}$ containing $\zeta$ into $\partial \mathbb{D}$, and recall that by Proposition 5.3.6 $\sigma$ has order of contact $n$ at $\zeta$. Thus, by Proposition 5.3.7 both $\sigma \circ \varphi \circ \psi$ and $\varphi \circ \sigma \circ \psi$ have order of contact $n$ at $\zeta$. Note also that $\sigma \circ \varphi \circ \psi$ and $\varphi \circ \sigma \circ \psi$ are is in the class $\mathcal{A}$ with $F=\{\zeta\}$. Therefore, by Theorem 4.2.1,

$$
C_{\sigma \circ \varphi \circ \psi}-C_{\varphi \circ \sigma \circ \psi} \in \mathcal{K} \Longleftrightarrow D_{n}(\sigma \circ \varphi \circ \psi, \zeta)=D_{n}(\varphi \circ \sigma \circ \psi, \zeta)
$$

$(2) \Longleftrightarrow(3)$ : Recall that as a consequence of Theorem 5.3.1, if $g$ is analytic at $z$ and $f$ is analytic at $g(z)$ then $D_{k}(g, z)$ and $D_{k}(f, g(z))$ determine $D_{k}(f \circ g, z)$. Since $\psi$ is invertible in a neighborhood of $\zeta$, we get that

$$
D_{n}(\sigma \circ \varphi \circ \psi, \zeta)=D_{n}(\varphi \circ \sigma \circ \psi, \zeta) \Longleftrightarrow D_{n}(\sigma \circ \varphi, \zeta)=D_{n}(\varphi \circ \sigma, \zeta)
$$

$(3) \Longleftrightarrow(4)$ : By Lemma 5.3.5, the $n$ 'th order data for $\sigma \circ \varphi$ and $\varphi \circ \sigma$ is given by

$$
\begin{aligned}
& D_{n}(\sigma \circ \varphi, \zeta)=\left(\zeta, 1,0, \ldots, 0, c \cdot \sigma^{\prime}(\zeta)\right) \\
& D_{n}(\varphi \circ \sigma, \zeta)=\left(\zeta, 1,0, \ldots, 0, c \cdot \sigma^{\prime}(\zeta)^{n}\right),
\end{aligned}
$$

where $c$ is a non zero constant. Hence the $n$ 'th order data is equal if and only if $\sigma^{\prime}(\zeta)=\sigma^{\prime}(\zeta)^{n}$. Recall that $\sigma^{\prime}(\zeta)=\frac{1}{\varphi_{e}^{\prime}(\zeta)}=\frac{1}{\varphi^{\prime}(\zeta)}$, and that $\varphi^{\prime}(\zeta)>0$ since $\varphi$ fixes $\zeta$. Thus $\sigma^{\prime}(\zeta)=\sigma^{\prime}(\zeta)^{n}$ if and only if $\varphi^{\prime}(\zeta)=1$. 
If $C_{\psi}$ were bounded below, compactness of $C_{\psi} A$ would imply compactness of $A$ for any operator $A$. We prove a somewhat weaker result using a technique from $[10$, section 4]. We denote the characteristic function on $\partial \mathbb{D}$ for the $\operatorname{arc} \Gamma_{\delta}=\{|z-\zeta|<$ $\delta\} \cap \partial \mathbb{D}$ by $\chi_{\delta}$.

Lemma 6.1.2. Let $\epsilon>0$ and $\psi=\psi_{\zeta, \epsilon}$. Then for any $\chi=\chi_{\delta}$ with $0<\delta<\epsilon$, and any operator $A$ we have

$$
C_{\psi} A \text { is compact } \Longrightarrow T_{\chi} A \text { is compact. }
$$

Proof. Suppose that $C_{\psi} A$ is compact. It suffices to show that the $H^{2}$ to $L^{2}$ operator $M_{\chi} A$ is compact. Let $t_{1}, t_{2}$ be such that $e^{i t}$ parametrizes the curve $\Gamma_{\delta}$ for $t_{1} \leq t \leq t_{2}$, and let $\alpha_{1}, \alpha_{2}$ be such that $\psi\left(e^{i t}\right)$ parametrizes the curve $\Gamma_{\delta}$ for $\alpha_{1} \leq t \leq \alpha_{2}$. Then

$$
\begin{aligned}
\left\|M_{\chi} f\right\|_{L_{2}} & =\int_{t_{1}}^{t_{2}}\left|f\left(e^{i \theta}\right)\right|^{2} \frac{d \theta}{2 \pi}=\int_{\Gamma_{\delta}} \frac{\left|f\left(e^{i \theta}\right)\right|^{2}}{2 \pi i z} d z=\int_{\alpha_{1}}^{\alpha_{2}}\left|f\left(\psi\left(e^{i t}\right)\right)\right|^{2}\left|\psi^{\prime}\left(e^{i t}\right)\right| \frac{d t}{2 \pi} \\
& \leq M \int_{\alpha_{1}}^{\alpha_{2}}\left|f\left(\psi\left(e^{i t}\right)\right)\right|^{2} \frac{d t}{2 \pi} \leq M\left\|C_{\psi} f\right\|_{L^{2}}^{2},
\end{aligned}
$$

where $M=\max \left\{\psi^{\prime}\left(e^{i t}\right): \alpha_{1} \leq t \leq \alpha_{2}\right\}$ is finite since $\psi$ can be analytically extended to a neighborhood of $\Gamma$.

Note that $C_{\psi} A$ is compact from $H^{2}$ to $H^{2}$ and so it is compact from $H^{2}$ to $L^{2}$, and that for all $f \in H^{2}$ we have that $\left\|M_{\chi} A f\right\|_{L_{2}} \leq \sqrt{M}\left\|C_{\psi} A f\right\|_{L_{2}}$. Thus $M_{\chi} A$ is compact from $H^{2}$ to $L^{2}$.

Theorem 6.1.3. Suppose $\varphi$ is a basic function with contact at $\zeta$ which fixes $\zeta$. Then $C_{\varphi}$ is essentially normal if and only if $\varphi^{\prime}(\zeta)=1$. 
Proof. By Proposition 6.1.1, there exists $\epsilon>0$ such that for $\psi=\psi_{\zeta, \epsilon}$, we have $C_{\psi}\left[C_{\varphi}, C_{\varphi}^{*}\right]$ is compact if and only if $\varphi^{\prime}(\zeta)=1$. Thus, it suffices to show that compactness of $C_{\psi}\left[C_{\varphi}, C_{\varphi}^{*}\right]$ implies compactness of $\left[C_{\varphi}, C_{\varphi}^{*}\right]$.

Suppose that $C_{\psi}\left[C_{\varphi}, C_{\varphi}^{*}\right]$ is compact. Then by Lemma 6.1 .2 with $\delta=\epsilon / 2$ and $\chi=\chi_{\delta}$, we get that $T_{\chi}\left[C_{\varphi}, C_{\varphi}^{*}\right]$ is compact. Now since $\varphi \in \mathcal{A}$ with $F=\{\zeta\}$ and $\chi$ is continuously differentiable at $\zeta$, by Corollary 4.3.2 and Proposition 4.3.5,

$$
T_{\chi} C_{\varphi} \equiv \chi(\zeta) C_{\varphi} \equiv C_{\varphi} \quad(\bmod \mathcal{K}) \quad \text { and } \quad T_{\chi} C_{\varphi}^{*} \equiv \chi(\varphi(\zeta)) C_{\varphi}^{*} \equiv C_{\varphi}^{*} \quad(\bmod \mathcal{K})
$$

Therefore,

$$
T_{\chi}\left[C_{\varphi}, C_{\varphi}^{*}\right] \equiv T_{\chi} C_{\varphi} C_{\varphi}^{*}-T_{\chi} C_{\varphi}^{*} C_{\varphi} \equiv\left[C_{\varphi}, C_{\varphi}^{*}\right] \quad(\bmod \mathcal{K})
$$

and so $\left[C_{\varphi}, C_{\varphi}^{*}\right]$ is compact as well.

\subsection{General Essential Normality}

In this section we will identify the essentially normal composition operators induced by a general function in the class $\mathcal{A}$. We let $\varphi$ be in $\mathcal{A}$ and denote $F(\varphi)=\left\{\zeta_{1}, \ldots, \zeta_{r}\right\}$ and the order of contact of $\varphi$ with $\partial \mathbb{D}$ at $\zeta_{j}$ by $n_{j}$. Then by Theorem 4.2 .5 , there exists a decomposition,

$$
C_{\varphi} \equiv C_{\varphi_{1}}+\ldots+C_{\varphi_{r}} \quad(\bmod \mathcal{K})
$$

where $\varphi_{j}$ is a basic function which has contact at $\zeta_{j}$ and satisfies

$$
D_{n_{j}}\left(\varphi_{j}, \zeta_{j}\right)=D_{n_{j}}\left(\varphi, \zeta_{j}\right)
$$


For each $j$, we denote $\lambda_{j}:=\varphi\left(\zeta_{j}\right)$, and let $\sigma_{j}$ be the unique branch of $\left(\varphi_{j}\right)_{e}^{-1}$ defined in some neighborhood of $\lambda_{j}$ which maps $\lambda_{j}$ to $\zeta_{j}$.

Lemma 6.2.1. Let $\varphi_{1}$ and $\varphi_{2}$ be basic functions with contact at $\zeta_{1}$ and $\zeta_{2}$ respectively, and denote $\lambda_{1}=\varphi_{1}\left(\zeta_{1}\right)$ and $\lambda_{2}=\varphi_{2}\left(\zeta_{2}\right)$. Then the following holds:

1. If $\zeta_{1} \neq \zeta_{2}$ then $C_{\varphi_{1}}^{*} C_{\varphi_{2}} \equiv 0 \quad(\bmod \mathcal{K})$

2. If $\lambda_{1} \neq \lambda_{2}$ then $C_{\varphi_{2}} C_{\varphi_{1}}^{*} \equiv 0 \quad(\bmod \mathcal{K})$

Proof. For the first part, suppose that $\zeta_{1} \neq \zeta_{2}$ and let $b$ be defined by $b(z)=\frac{z-\zeta_{1}}{\zeta_{2}-\zeta_{1}}$, so that $b$ is continuous at $\zeta_{1}$ and $\zeta_{2}$ and satisfies $b\left(\zeta_{1}\right)=0$ and $b\left(\zeta_{2}\right)=1$. Then by Corollary 4.3.2,

$$
T_{b} C_{\varphi_{2}} \equiv b\left(\zeta_{2}\right) C_{\varphi_{2}} \equiv C_{\varphi_{2}} \quad(\bmod \mathcal{K}), \quad \text { and } \quad T_{\bar{b}} C_{\varphi_{1}} \equiv \overline{b\left(\zeta_{1}\right)} C_{\varphi_{1}} \equiv 0 \quad(\bmod \mathcal{K})
$$

and combining these two equations, we get

$$
C_{\varphi_{1}}^{*} C_{\varphi_{2}} \equiv C_{\varphi_{1}}^{*}\left(T_{b} C_{\varphi_{2}}\right) \equiv C_{\varphi_{1}}^{*} T_{\bar{b}}^{*} C_{\varphi_{2}} \equiv\left(T_{\bar{b}} C_{\varphi_{1}}\right)^{*} C_{\varphi_{2}} \equiv 0 \quad(\bmod \mathcal{K})
$$

For the second part, suppose $\lambda_{1} \neq \lambda_{2}$ and let $c$ be defined by $c(z)=\frac{z-\lambda_{2}}{\lambda_{1}-\lambda_{2}}$, so that $c$ is continuously differentiable in neighborhoods of $\lambda_{1}$ and $\lambda_{2}$ and satisfies $c\left(\lambda_{1}\right)=1$ and $c\left(\lambda_{2}\right)=0$. Then by Proposition 4.3.5,

$$
T_{c} C_{\varphi_{1}}^{*} \equiv c\left(\lambda_{1}\right) C_{\varphi_{1}}^{*} \equiv C_{\varphi_{1}}^{*} \quad(\bmod \mathcal{K}), \quad \text { and } \quad T_{\bar{c}} C_{\varphi_{2}}^{*} \equiv \overline{c\left(\lambda_{2}\right)} C_{\varphi_{2}}^{*} \equiv 0 \quad(\bmod \mathcal{K}),
$$

and combining these two equations, we get

$$
C_{\varphi_{2}} C_{\varphi_{1}}^{*} \equiv C_{\varphi_{2}}\left(T_{c} C_{\varphi_{1}}^{*}\right) \equiv\left(C_{\varphi_{2}}^{*}\right)^{*}\left(T_{\bar{c}}\right)^{*} C_{\varphi_{1}}^{*} \equiv\left(T_{\bar{c}} C_{\varphi_{2}}^{*}\right)^{*} C_{\varphi_{1}}^{*} \equiv 0 \quad(\bmod \mathcal{K}) .
$$


Proposition 6.2.2. Let $\varphi$ in $\mathcal{A}$ be such that $C_{\varphi}$ is essentially normal. Let $\zeta_{1}, \ldots, \zeta_{r}$, $n_{1}, \ldots, n_{r}, \lambda_{1}, \ldots, \lambda_{r}, \varphi_{1}, \ldots, \varphi_{r}$ and $\sigma_{1}, \ldots, \sigma_{r}$ be as described above. Then for any $i$ in $\{1, \ldots, r\}$ there exists a unique $j$ such that $\varphi\left(\zeta_{j}\right)=\zeta_{i}$. Furthermore, for this $j$, $n_{j}=n_{i}=n$ and

$$
D_{n}\left(\sigma_{i} \circ \varphi_{i}, \zeta_{i}\right)=D_{n}\left(\varphi_{j} \circ \sigma_{j}, \lambda_{j}\right) .
$$

Proof. By relabeling the functions, it suffices to show this for $i=1$. By Lemma 6.2.1, we see that

$$
\begin{aligned}
{\left[C_{\varphi}, C_{\varphi}^{*}\right] } & \equiv\left[C_{\varphi_{1}}+\ldots+C_{\varphi_{r}}, C_{\varphi_{1}}^{*}+\ldots+C_{\varphi_{r}}^{*}\right] \\
& \equiv \sum_{j, k=1}^{r} C_{\varphi_{k}} C_{\varphi_{j}}^{*}-\sum_{j, k=1}^{r} C_{\varphi_{j}}^{*} C_{\varphi_{k}} \equiv \sum_{\substack{j, k=1, \ldots, r \\
\lambda_{k}=\lambda_{j}}} C_{\varphi_{k}} C_{\varphi_{j}}^{*}-\sum_{j=1}^{r} C_{\varphi_{j}}^{*} C_{\varphi_{j}} \quad(\bmod \mathcal{K}) .
\end{aligned}
$$

Now let $b$ be a polynomial such that $b\left(\zeta_{1}\right)=1$ and $b$ is 0 at all the points in $\left\{\zeta_{2}, \ldots \zeta_{r}, \lambda_{1}, \ldots, \lambda_{r}\right\} \backslash\left\{\zeta_{1}\right\}$. Then $b$ is continuously differentiable everywhere and so by Corollary 4.3.2 and Proposition 4.3.5,

$$
\begin{aligned}
& M_{b} C_{\varphi_{1}} \equiv C_{\varphi_{1}} \quad(\bmod \mathcal{K}), \quad M_{b} C_{\varphi_{i}} \equiv 0 \quad(\bmod \mathcal{K}) \quad \text { for } i \neq 1, \\
& M_{b} C_{\varphi_{i}}^{*} \equiv C_{\varphi_{i}}^{*} \quad(\bmod \mathcal{K}) \quad \text { if } \lambda_{i}=\zeta_{1}, \quad M_{b} C_{\varphi_{i}}^{*} \equiv 0 \quad(\bmod \mathcal{K}) \quad \text { if } \lambda_{i} \neq \zeta_{1} .
\end{aligned}
$$

Therefore, we have

$$
\begin{aligned}
M_{b}\left[C_{\varphi}, C_{\varphi}^{*}\right] & \equiv \sum_{\substack{j, k=1 \ldots r \\
\lambda_{j}=\lambda_{k}}} M_{b} C_{\varphi_{k}} C_{\varphi_{j}}^{*}-\sum_{j=1}^{r} M_{b} C_{\varphi_{j}}^{*} C_{\varphi_{j}} \\
& \equiv \sum_{\substack{j=1 \ldots r \\
\lambda_{j}=\lambda_{1}}} C_{\varphi_{1}} C_{\varphi_{j}}^{*}-\sum_{\substack{j=1 \ldots r \\
\lambda_{j}=\zeta_{1}}} C_{\varphi_{j}}^{*} C_{\varphi_{j}}(\bmod \mathcal{K})
\end{aligned}
$$


By Proposition 5.2.3, for each $\varphi_{j}$ there exists a neighborhood $W_{j}$ of $\lambda_{j}$ such that

$$
C_{\psi} C_{\varphi_{j}}^{*} \equiv \frac{1}{\left|\varphi_{j}^{\prime}\left(\zeta_{j}\right)\right|} C_{\sigma_{j} \circ \psi} \quad(\bmod \mathcal{K})
$$

for any self-map $\psi$ of $\mathbb{D}$ that is analytic at $\lambda_{j}$, satisfies $\psi^{-1}\left(\left\{\lambda_{j}\right\}\right)=\left\{\lambda_{j}\right\}$ and maps $\mathbb{D}$ into $W_{j}$.

Notice that if $\lambda_{j}=\zeta_{1}$ then $W_{j}$ is a neighborhood of $\zeta_{1}$ and if $\lambda_{j}=\lambda_{1}$ then $\lambda_{1} \in W_{j}$ and so that $\varphi_{1}^{-1}\left(W_{j}\right)$ is a neighborhood of $\zeta_{1}$. We can therefore define a neighborhood $W$ of $\zeta_{1}$ by

$$
W=\left(\bigcap_{\lambda_{j}=\lambda_{1}} \varphi_{1}^{-1}\left(W_{j}\right)\right) \cap\left(\bigcap_{\lambda_{j}=\zeta_{1}} W_{j}\right) .
$$

Now let $\epsilon>0$ be such that $W_{0}:=\left\{\left|z-\zeta_{1}\right| \leq \epsilon\right\} \cap \mathbb{D}$ is contained in $W$, and choose $\psi=\psi_{\zeta_{1}, \epsilon}$.

Now for $j$ such that $\lambda_{j}=\lambda_{1}$, we see that $\varphi_{1} \circ \psi$ maps $\overline{\mathbb{D}}$ into $\varphi_{1}(W) \subset W_{j}$ and so by Proposition 5.2.3,

$$
C_{\psi} C_{\varphi_{1}} C_{\varphi_{j}}^{*} \equiv C_{\varphi_{1} \circ \psi} C_{\varphi_{j}}^{*} \equiv \frac{1}{\left|\varphi_{j}^{\prime}\left(\zeta_{j}\right)\right|} C_{\sigma_{j} \circ \varphi_{1} \circ \psi} \quad(\bmod \mathcal{K}) .
$$

Similarly, for $j$ such that $\lambda_{j}=\zeta_{1}$, we see that $\psi$ maps $\overline{\mathbb{D}}$ into $V \subset W_{j}$ so by Proposition 5.2.3,

$$
C_{\psi} C_{\varphi_{j}}^{*} C_{\varphi_{j}} \equiv \frac{1}{\left|\varphi_{j}^{\prime}\left(\zeta_{j}\right)\right|} C_{\sigma_{j} \circ \psi} C_{\varphi_{j}} \equiv \frac{1}{\left|\varphi_{j}^{\prime}\left(\zeta_{j}\right)\right|} C_{\varphi_{j} \circ \sigma_{j} \circ \psi} \quad(\bmod \mathcal{K}) .
$$

By our assumption that $C_{\varphi}$ is essentially normal, the operator $C_{\psi} M_{b}\left[C_{\varphi}, C_{\varphi}^{*}\right]$ is compact. We can now express this operator as a linear combination of composition 
operators by

$$
\begin{aligned}
0 \equiv C_{\psi} M_{b}\left[C_{\varphi}, C_{\varphi}^{*}\right] & \equiv \sum_{\substack{j=1 \ldots r \\
\lambda_{j}=\lambda_{1}}} C_{\psi} C_{\varphi_{1}} C_{\varphi_{j}}^{*}-\sum_{\substack{j=1 \ldots r \\
\lambda_{j}=\zeta_{1}}} C_{\psi} C_{\varphi_{j}}^{*} C_{\varphi_{j}} \\
& \equiv \sum_{\lambda_{k}=\lambda_{1}} \frac{1}{\left|\varphi^{\prime}\left(\zeta_{k}\right)\right|} C_{\sigma_{k} \circ \varphi_{1} \circ \psi}-\sum_{\lambda_{j}=\zeta_{1}} \frac{1}{\left|\varphi^{\prime}\left(\zeta_{j}\right)\right|} C_{\varphi_{j} \circ \sigma_{j} \circ \psi} \quad(\bmod \mathcal{K})
\end{aligned}
$$

In order to draw some conclusions from Theorem 4.2.1, we discuss some properties of the inducing maps above. First note that $\psi$ maps an arc of $\partial \mathbb{D}$ containing $\zeta_{1}$ to an $\operatorname{arc}$ of $\partial \mathbb{D}$, and recall that by Proposition 5.3.6 $\sigma_{j}$ has order of contact $n_{j}$ at $\lambda_{j}=\lambda_{1}$. Thus, by Proposition 5.3.7, $\sigma_{j} \circ \varphi_{1} \circ \psi$ has order of contact $\min \left(n_{1}, n_{j}\right)$ at $\zeta_{1}$. We also note that $\sigma_{j} \circ \varphi_{1} \circ \psi$ is in the class $\mathcal{A}$ with $F\left(\sigma_{j} \circ \varphi_{1} \circ \psi\right)=\left\{\zeta_{1}\right\}$ and maps $\zeta_{1}$ to $\zeta_{j}$ (indeed $\zeta_{1} \stackrel{\psi}{\longmapsto} \zeta_{1} \stackrel{\varphi_{1}}{\longmapsto} \lambda_{1}=\lambda_{j} \stackrel{\sigma_{j}}{\longmapsto} \zeta_{j}$ ). Similarly, by Proposition 5.3.7, $\varphi_{j} \circ \sigma_{j} \circ \psi$ has order of contact $n_{j}$ at $\zeta_{1}$, and $\varphi_{j} \circ \sigma_{j} \circ \psi$ is in the class $\mathcal{A}$ with $F\left(\varphi_{j} \circ \sigma_{j} \circ \psi\right)=\left\{\zeta_{1}\right\}$ and fixes $\zeta_{1}$ (indeed $\zeta_{1} \stackrel{\psi}{\longmapsto} \zeta_{1}=\lambda_{j} \stackrel{\sigma_{j}}{\longmapsto} \zeta_{j} \stackrel{\varphi_{j}}{\longmapsto} \lambda_{j}=\zeta_{1}$ ).

Thus, all the inducing maps above are in $\mathcal{A}$ with $F=\left\{\zeta_{1}\right\}$, and each inducing map in the first sum maps $\zeta_{1}$ to $\zeta_{j}$, while all inducing maps in the second sum fix $\zeta_{1}$. Since the $\zeta_{j}$ 's are distinct, any term in the first sum originating from $j \neq 1$ would prevent compactness by Theorem 4.2.1. Thus for all $j \neq 1$ we must have that $\lambda_{j} \neq \lambda_{1}$ and so Eq. (6.2.1) becomes

$$
0 \equiv \frac{1}{\left|\varphi^{\prime}\left(\zeta_{1}\right)\right|} C_{\sigma_{1} \circ \varphi_{1} \circ \psi}-\sum_{\lambda_{j}=\zeta_{1}} \frac{1}{\left|\varphi^{\prime}\left(\zeta_{j}\right)\right|} C_{\varphi_{j} \circ \sigma_{j} \circ \psi} \quad(\bmod \mathcal{K}) .
$$

Note that by symmetry $\lambda_{1}, \ldots, \lambda_{r}$ are distinct, and so there is at most one $j$ such that $\lambda_{j}=\zeta_{1}$. Thus, the second sum contains at most one term. Again by Theorem 4.2.1, 
the second sum cannot be empty, so there is a unique $j$ such that $\lambda_{j}=\zeta_{1}$. Furthermore, for this $j,\left|\varphi^{\prime}\left(\zeta_{1}\right)\right|=\left|\varphi^{\prime}\left(\zeta_{j}\right)\right|$ and $\sigma_{1} \circ \varphi_{1} \circ \psi$ and $\varphi_{j} \circ \sigma_{j} \circ \psi$ have equal order of contact, $n=n_{1}=n_{j}$, at $\zeta_{1}$ and

$$
D_{n}\left(\sigma_{1} \circ \varphi_{1} \circ \psi, \zeta_{1}\right)=D_{n}\left(\varphi_{j} \circ \sigma_{j} \circ \psi, \zeta_{1}\right)
$$

Recall that as a consequence of Theorem 5.3.1, if $g$ is analytic at $z$ and $f$ is analytic at $g(z)$ then $D_{k}(g, z)$ and $D_{k}(f, g(z))$ determine $D_{k}(f \circ g, z)$. Since $\psi$ is invertible in a neighborhood of $\zeta$, we get that

$$
D_{n}\left(\sigma_{1} \circ \varphi_{1}, \zeta_{1}\right)=D_{n}\left(\varphi_{j} \circ \sigma_{j}, \zeta_{1}\right)
$$

Lemma 6.2.3. Let $\varphi$ be in $\mathcal{A}$ be such that $C_{\varphi}$ is essentially normal and suppose that $\varphi$ fixes $\zeta \in F(\varphi)$. Then $\zeta$ is the unique Denjoy-Wolff point of $\varphi$.

Proof. We label the points of $F(\varphi)$ so that $\zeta_{1}=\zeta$ and let $\varphi_{1}$ and $\sigma_{1}$ be as described above. Then by Proposition 6.2.2,

$$
D_{n}\left(\sigma_{1} \circ \varphi_{1}, \zeta_{1}\right)=D_{n}\left(\varphi_{1} \circ \sigma_{1}, \zeta_{1}\right)
$$

In particular, the $n$th derivatives are equal and so by Lemma 5.3.5,

$$
c \cdot \sigma_{1}^{\prime}\left(\zeta_{1}\right)=c \cdot \sigma_{1}^{\prime}\left(\zeta_{1}\right)^{n}
$$

where $c \neq 0$. Using the fact that $\sigma_{1}^{\prime}\left(\zeta_{1}\right)=1 / \varphi_{1}^{\prime}\left(\zeta_{1}\right)>0$, we get that

$$
\varphi^{\prime}\left(\zeta_{1}\right)=\varphi_{1}^{\prime}\left(\zeta_{1}\right)=1
$$

and so, by Theorem 2.3.6, $\zeta_{1}$ is the unique Denjoy-Wolff point of $\varphi$. 
Theorem 6.2.4. Let $\varphi$ be in $\mathcal{A}$ with $F(\varphi)=\left\{\zeta_{1}, \ldots, \zeta_{r}\right\}$. Then $C_{\varphi}$ is essentially normal if and only if $r=1, \varphi$ fixes $\zeta_{1}$ and $\varphi^{\prime}\left(\zeta_{1}\right)=1$.

Proof. If $r=1, \varphi$ fixes $\zeta_{1}$ and $\varphi^{\prime}\left(\zeta_{1}\right)=1$ then $\left[C_{\varphi}, C_{\varphi}^{*}\right] \equiv\left[C_{\varphi_{1}}, C_{\varphi_{1}}^{*}\right]$ is compact by Theorem 6.1.3.

For the other direction, we suppose that $C_{\varphi}$ is essentially normal. In order to obtain a contradiction, suppose that $r>1$. By Lemma 6.2.3, $F(\varphi)$ contains at most one fixed point, so there exists some index $i$ such that $\zeta_{i} \in F(\varphi)$ is not fixed.

Recall that by Proposition 6.2.2, for each $i \in\{1, \ldots, r\}$ there is a unique $j$ such that $\varphi\left(\zeta_{j}\right)=\zeta_{i}$. Let $i_{1}=i$ and let $i_{2}$ be the unique index for $i_{1}$ as above. Continue to define $i_{l+1}$ as the unique index for $i_{l}$ as above until a cycle is closed, $i_{1} \rightarrow \ldots \rightarrow i_{k} \rightarrow i_{1}$.

Let $\varphi_{(k)}=\varphi \circ \ldots \circ \varphi$ and note that the operator $C_{\varphi_{(k)}}=C_{\varphi}^{k}$ is essentially normal since $C_{\varphi}$ and $C_{\varphi}^{*}$ commute modulo $\mathcal{K}$. We get that $\varphi_{(k)} \in \mathcal{A}$ induces an essentially normal composition operator and fixes $k>1$ distinct points, $\zeta_{i_{1}}, \ldots, \zeta_{i_{k}}$, in $F\left(\varphi_{(k)}\right)$. This is a contradiction by Lemma 6.2 .3 , and so we must have that $r=1$. By Theorem 6.1.3 we get that $\varphi$ fixes $\zeta_{1}$ and $\varphi^{\prime}\left(\zeta_{1}\right)=1$.

Corollary 6.2.5. Let $\varphi$ be a self-map of $\mathbb{D}$ which extends analytically to a neighborhood of $\mathbb{D}$ and that is not a finite Blaschke product. Then $C_{\varphi}$ is non-trivially essentially normal if and only if there exists $\zeta \in \partial \mathbb{D}$ such that $\varphi$ fixes $\zeta$ and $\varphi^{\prime}(\zeta)=1$, and $\varphi$ maps $\partial \mathbb{D} \backslash\{\zeta\}$ into $\mathbb{D}$.

Proof. By Proposition 4.1.1, $\varphi \in \mathcal{A}$. If $F(\varphi)$ is empty then $\|\varphi\|_{\infty}<1$ and so $C_{\varphi} \in \mathcal{K}$ is trivially essentially normal. Otherwise, the claim follows from Theorem 6.2.4. 


\subsection{Construction of Essentially Normal Composi-}

\section{tion Operators}

We combine the criterion for essential normality given in Theorem 6.2.4 with several results from Chapter 3 to construct essentially normal composition operators which have arbitrary even order of contact with $\partial \mathbb{D}$ at a point $\zeta$.

Proposition 6.3.1. Let $\zeta \in \partial \mathbb{D}, n=2 m$ and

$$
s_{1}, \ldots, s_{m-1} \in \mathbb{R}, \quad t_{1}, \ldots, t_{m-1}>0, \quad w \in \mathbb{H}
$$

Let $\varphi$ be defined by $\varphi=\tau_{\zeta}^{-1} \circ f \circ \tau_{\zeta}$ where

$$
f(z)=L\left[A(0,1)(z) A\left(s_{1}, t_{1}\right)(z) \cdots A\left(s_{m-1}, t_{m-1}\right)(z)\right] w
$$

and

$$
A(s, t)(z)=\left[\begin{array}{cc}
s t z & -s-t z \\
t z & -1
\end{array}\right] .
$$

Then $\varphi$ is a degree $m$ rational function with order of contact $n$ at $\zeta$ which induces an essentially normal composition operator.

Proof. By Proposition 3.3.3, $f$ has order of contact $n$ with $\mathbb{R}$ at $z=0$, and so $\varphi$ has order of contact $n$ with $\partial \mathbb{D}$ at $\zeta$. Note that $f$ is obtained by taking $m$ augmentations of the constant function $g(z) \equiv w$. By Proposition 3.4.1 and Proposition 3.4.2, $f$ is a rational function of degree $m$ mapping $\widehat{\mathbb{R}} \backslash\{0\}$ into $\mathbb{H}$. Since the last augmentation performed to obtain $f$ has parameters $a_{0}=0, a_{1}=1, f$ satisfies $f(0)=0, f^{\prime}(0)=1$. 
Note that $\tau_{\zeta}$ preserves the degree of a rational function, and so $\varphi=\tau_{\zeta}^{-1} \circ f \circ \tau_{\zeta}$ is a degree $m$ rational function. Additionally, the properties of $f$ ensure that $\varphi$ is in $\mathcal{A}$ with $F(\varphi)=\{\zeta\}$ and $\varphi$ fixes $\zeta$ and satisfies $\varphi^{\prime}(\zeta)=1$. Thus, by Theorem 6.2.4, the operator $C_{\varphi}$ is essentially normal.

For order of contact $n=4$ we define $f(s, t, w)$ and $\varphi(\zeta, s, t, w)$ for any $s \in \mathbb{R}$, $t>0$ and $w \in \mathbb{H}$ and $\zeta \in \partial \mathbb{D}$ by

$$
\begin{aligned}
f(s, t, w)(z) & =L[A(0,1)(z) A(s, t)(z)] w \\
& =L\left[\left[\begin{array}{cc}
0 & -z \\
z & -1
\end{array}\right]\left[\begin{array}{cc}
s z & -s-t z \\
t z & -1
\end{array}\right]\right] w=\frac{-t z^{2} w+z}{\left(s t z^{2}-t z\right) w-s z-t z^{2}+1},
\end{aligned}
$$

and

$$
\varphi(\zeta, s, t, w)=\tau_{\zeta}^{-1} \circ f(s, t, w) \circ \tau_{\zeta}
$$

Then $\varphi(\zeta, s, t, w)$ has order of contact 4 with $\partial \mathbb{D}$ at $\zeta$ and induces an essentially normal composition operator.

We examine the order of contact 2 case for motivation. It is known that for a linear fractional $\varphi$ the operator $C_{\varphi}$ is non-trivially essentially normal if and only if $\varphi$ is a parabolic non-automorphisms [6]. In this case, $\varphi$ corresponds to a translation in the right half-plane.

We transfer the contact 2 version of the formula above to the right half-plane using the conformal mapping $T(z)=\frac{i}{z}$ which maps $\mathbb{H}$ to the right half-plane and 0 
to $\infty$. For any $w \in \mathbb{H}$ and $\zeta \in \partial \mathbb{D}$ the self-map of $\mathbb{H}$

$$
f_{w}(z)=L[A(0,1)(z)] w=L\left[\begin{array}{cc}
0 & -z \\
z & -1
\end{array}\right] w=\frac{-z}{w z-1}=\frac{1}{\frac{1}{z}-w}
$$

corresponds to the self map of the right half-plane

$$
\left(T \circ f_{w} \circ T\right)(z)=\frac{i}{f_{w}(i / z)}=i\left(\frac{z}{i}-w\right)=z-i w
$$

Applying this same procedure to our order of contact 4 functions

$$
\begin{aligned}
f(s, t, w)(z) & =\frac{-t z^{2} w+z}{\left(s t z^{2}-t z\right) w-s z-t z^{2}+1} \\
= & \frac{(1-t z w) z}{(w t z-1) s z+(1-t w z)-t z^{2}} \\
= & \frac{1}{-s+\frac{1}{z}-\frac{t z}{1-t w z}}=\frac{1}{-s+\frac{1}{z}+\frac{1}{w-\frac{1}{t z}}},
\end{aligned}
$$

we calculate

$$
\begin{aligned}
(T \circ f(s, t, w) \circ T)(z) & =\frac{i}{f(s, t, w)(i / z)}=i\left(-s+\frac{z}{i}+\frac{1}{w-\frac{z}{i t}}\right) \\
& =z-i s+\frac{1}{\frac{1}{t} z-i w} .
\end{aligned}
$$

In Fig. 6.1, we sketch the image of $\partial \mathbb{D}$ under $\varphi=\varphi(1, s, t, w)$ for various parameters. 
Figure 6.1: Examples of inducing maps of essentially normal composition operators

(a) $\varphi(1,0,1, i)=\frac{z^{2}+2 z+1}{z^{2}-2 z+5}$

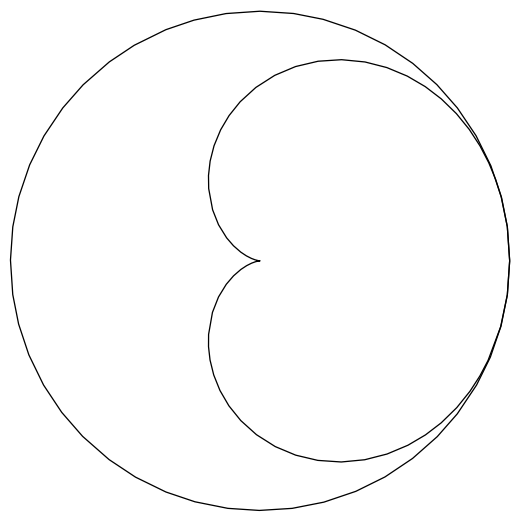

(c) $\varphi(1,0,1 / 2, i)=\frac{z+1}{z^{2}-3 z+4}$

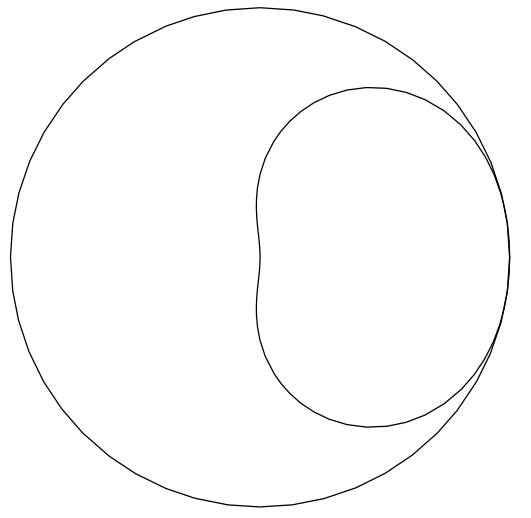

(e) $\varphi(1,0,1,2 i)=\frac{-z^{2}+4 z+1}{z^{2}-4 z+7}$

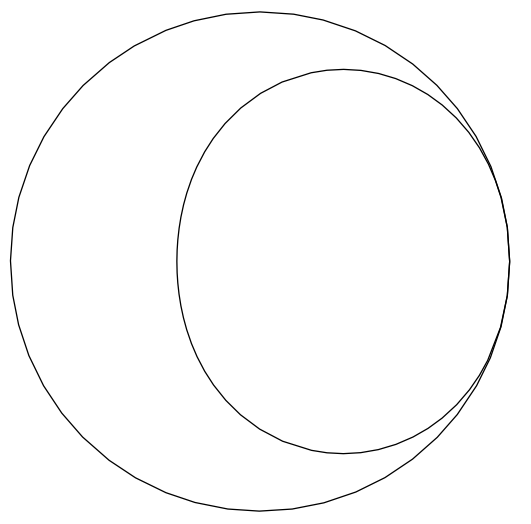

(b) $\varphi(1,1,1, i)=\frac{z^{2}+2(1+i) z+1-2 i}{z^{2}-2(1-i) z+5-2 i}$

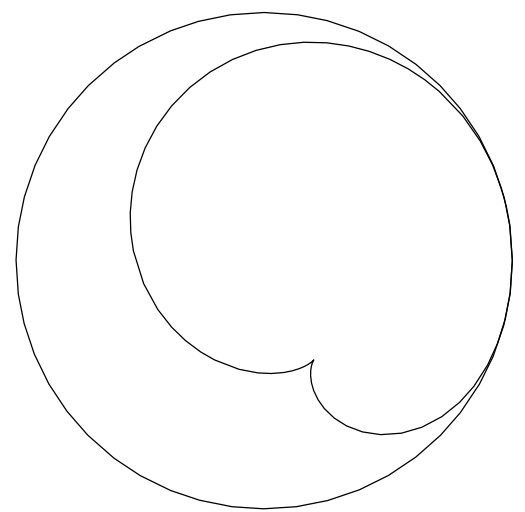

(d) $\varphi(1,0,2, i)=\frac{z^{2}+7}{3 z^{2}+4 z+1}$

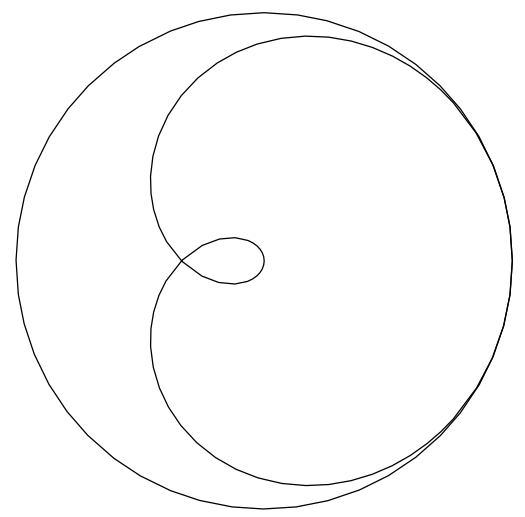

(f) $\varphi(1,0,1, i / 2+1)=\frac{(-2+2 i) z^{2}+(2+i) z+i}{i z^{2}-(4+2 i) z+2+4 i}$

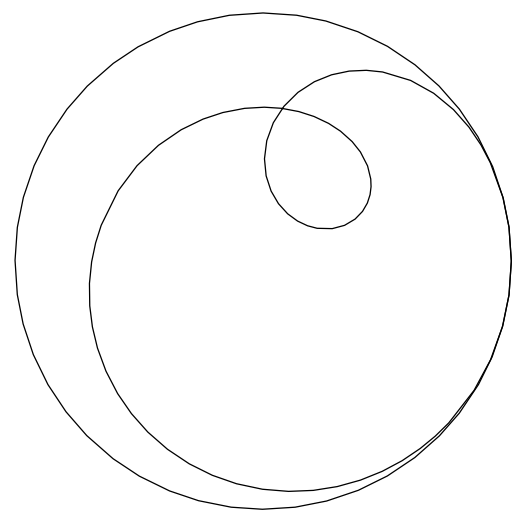




\section{Bibliography}

[1] Jim Agler, Zinaida A. Lykova, and N. J. Young. The boundary CarathéodoryFejér interpolation problem. J. Math. Anal. Appl., 382(2):645-662, 2011.

[2] Jim Agler, Zinaida A. Lykova, and N. J. Young. Pseudo-Taylor expansions and the Carathéodory-Fejér problem. J. Math. Anal. Appl., 386(1):308-318, 2012.

[3] Jim Agler and N. J. Young. Boundary Nevanlinna-Pick interpolation via reduction and augmentation. Math. Z., 268(3-4):791-817, 2011.

[4] William Arveson. A short course on spectral theory, volume 209 of Graduate Texts in Mathematics. Springer-Verlag, New York, 2002.

[5] Joseph A. Ball, Israel Gohberg, and Leiba Rodman. Interpolation of rational matrix functions, volume 45 of Operator Theory: Advances and Applications. Birkhäuser Verlag, Basel, 1990.

[6] Paul S. Bourdon, David Levi, Sivaram K. Narayan, and Joel H. Shapiro. Which linear-fractional composition operators are essentially normal? J. Math. Anal. 
Appl., 280(1):30-53, 2003.

[7] Paul S. Bourdon and Joel H. Shapiro. Adjoints of rationally induced composition operators. J. Funct. Anal., 255(8):1995-2012, 2008.

[8] C. Caratheodory and L. Fejèr. ber den zusammenhang der extremen von harmonischen funktionen mit ihren koeffizienten und ber den picard-landauschen satz. Rendiconti del Circolo Matematico di Palermo, 32(1):218-239, 1911.

[9] Joseph A. Cima, Alec L. Matheson, and William T. Ross. The Cauchy transform, volume 125 of Mathematical Surveys and Monographs. American Mathematical Society, Providence, RI, 2006.

[10] Joseph A. Cima, James Thomson, and Warren Wogen. On some properties of composition operators. Indiana Univ. Math. J., 24:215-220, 1974/75.

[11] Carl C. Cowen. Linear fractional composition operators on $H^{2}$. Integral Equations Operator Theory, 11(2):151-160, 1988.

[12] Carl C. Cowen and Eva A. Gallardo-Gutiérrez. A new class of operators and a description of adjoints of composition operators. J. Funct. Anal., 238(2):447-462, 2006.

[13] Carl C. Cowen and Barbara D. MacCluer. Composition operators on spaces of analytic functions. Studies in Advanced Mathematics. CRC Press, Boca Raton, FL, 1995. 
[14] Stephen Fisher. Complex Variables. Dover, second edition, 1990.

[15] Christopher Hammond, Jennifer Moorhouse, and Marian E. Robbins. Adjoints of composition operators with rational symbol. J. Math. Anal. Appl., 341(1):626639, 2008.

[16] Kenneth Hoffman. Banach spaces of analytic functions. Prentice-Hall Series in Modern Analysis. Prentice-Hall Inc., Englewood Cliffs, N. J., 1962.

[17] Warren P. Johnson. The curious history of Faà di Bruno's formula. Amer. Math. Monthly, 109(3):217-234, 2002.

[18] Gaston Julia. Extension nouvelle d'un lemme de Schwarz. Acta Math., 42(1):349$355,1920$.

[19] Thomas Kriete and Jennifer Moorhouse. Linear relations in the Calkin algebra for composition operators. Trans. Amer. Math. Soc., 359(6):2915-2944 (electronic), 2007.

[20] Thomas L. Kriete, Barbara D. MacCluer, and Jennifer L. Moorhouse. Toeplitzcomposition $C^{*}$-algebras. J. Operator Theory, 58(1):135-156, 2007.

[21] Rubén A. Martínez-Avendaño and Peter Rosenthal. An introduction to operators on the Hardy-Hilbert space, volume 237 of Graduate Texts in Mathematics. Springer, New York, 2007. 
[22] Nikolai K. Nikolski. Operators, functions, and systems: an easy reading. Vol. 1, volume 92 of Mathematical Surveys and Monographs. American Mathematical Society, Providence, RI, 2002. Hardy, Hankel, and Toeplitz, Translated from the French by Andreas Hartmann.

[23] Gert K. Pedersen. Analysis now, volume 118 of Graduate Texts in Mathematics. Springer-Verlag, New York, 1989.

[24] Donald Sarason. Sub-Hardy Hilbert spaces in the unit disk. University of Arkansas Lecture Notes in the Mathematical Sciences, 10. John Wiley \& Sons Inc., New York, 1994. A Wiley-Interscience Publication.

[25] Joel H. Shapiro. Composition operators and classical function theory. Universitext: Tracts in Mathematics. Springer-Verlag, New York, 1993. 